JOURNAL OF INTEGRAL EQUATIONS

AND APPLICATIONS

Volume 3, Number 1, Winter 1991

\title{
STRAIN SOFTENING IN VISCOELASTICITY OF THE RATE TYPE
}

\author{
ATHANASIOS E. TZAVARAS \\ Dedicated to John A. Nohel on his 65th birthday.
}

1. Introduction. The intent of this article is to study the behavior of solutions $(v(x, t), u(x, t))$ of the system of differential equations

$$
\begin{aligned}
v_{t} & =\sigma_{x} \\
u_{t} & =v_{x},
\end{aligned}
$$

where

$$
\sigma=\tau(u) v_{x}^{n}
$$

with $\tau(u)$ a smooth function satisfying

$$
\tau(u)>0, \quad \tau^{\prime}(u)<0
$$

and $n$ a positive parameter. Equations (1.1)-(1.3) give rise to a coupled system of partial differential equations in one space dimension. They are supplemented with initial conditions

$$
v(x, 0)=v_{0}(x), \quad u(x, 0)=u_{0}(x),
$$

and, as a consequence,

$$
\sigma(x, 0)=\sigma_{0}(x):=\tau\left(u_{0}(x)\right) v_{0 x}^{n}(x) ;
$$

also, with boundary conditions that are discussed later.

To gain some perspective on the problem, note that, if $n=0$, (1.1)-(1.3) lead to the pair of conservation laws

$$
\begin{aligned}
& v_{t}=\tau(u)_{x} \\
& u_{t}=v_{x} .
\end{aligned}
$$

Research partially supported by the National Science Foundation under Grant No. DMS-8716132 and the Army Research Office under Contract No. DAAL03-88$\mathrm{K}-0185$.

Copyright (C)1991 Rocky Mountain Mathematics Consortium 
If $\tau^{\prime}(u)>0$, then (1.7) is hyperbolic; however, under (1.4) the system (1.7) is elliptic and the initial value problem is ill-posed. Nevertheless, it admits an interesting class of special solutions

$$
\begin{aligned}
& \bar{v}(x, t)=x \\
& \bar{u}(x, t)=t+u_{0},
\end{aligned}
$$

where $u_{0}$ is an arbitrary constant. Equations (1.1)-(1.3) with $n>0$ can be thought of as a particular regularization of (1.7).

A motivation for studying this problem stems from a program of understanding the phenomenon of shear band formation at high strain rates. Shear bands are narrow regions of intensely concentrated shearing deformation that are observed during the plastic deformation of many materials. The occurrence of shear bands is typically associated with strain softening type response, past a critical strain, of the measured average shear stress $\Sigma(t)$ versus the measured average shear strain $U(t)$; that is, $\Sigma=\tau(U)$, where $\tau(\cdot)$ is increasing up to a certain critical strain and decreasing thereafter. Various mechanisms and associated continuum thermomechanics models, often depending on the particular context, have been proposed for the explanation of shear bands (see Shawki and Clifton [15] for an excellent survey of the related literature). An underlying common feature of several models is that they are regularizations of an ill-posed problem or that some associated linearized problem exhibits growth of high-frequency modes.

The model employed here describes the plastic shearing of an infinite plate of unit thickness subjected to either prescribed tractions or prescribed velocities at the boundaries. In this framework, $v(x, t)$ describes the velocity field in the shearing direction, $\sigma(x, t)$ stands for the shear stress and $u(x, t)$ for the plastic shear strain. Equation (1.1) describes the balance of linear momentum, while (1.2) is a kinematic compatibility relation (note that elastic effects are neglected); (1.1) and (1.2) are taken over $(x, t) \in[0,1] \times\{t>0\}$ and are supplemented with the boundary conditions

$$
\sigma(0, t)=1, \quad \sigma(1, t)=1, \quad t>0,
$$

in case the shearing deformation is caused by prescribed tractions at the boundaries, or

$$
v(0, t)=0, \quad v(1, t)=1, \quad t>0,
$$


in the case of prescribed velocities. The constitutive law (1.3) is appropriate for a material exhibiting strain softening, as manifested in (1.4), and strain rate sensitivity, the strength of which is measured by the parameter $n$. Our objective is to use (1.1)-(1.6), (1.9) as a test problem to analyze the competition between the destabilizing effect of strain softening versus the stabilizing effect of strain rate dependence.

Technically, the model (1.1)-(1.3) belongs to the class of isothermal viscoelasticity of the rate type (for general information on the mathematical theory of viscoelasticity, the reader is referred to Renardy, Hrusa and Nohel [14]). Metals, in general, exhibit strain hardening in isothermal deformations. However, an increase in temperature causes a decrease in the yield stress, so that, in an adiabatic deformation, the combined effect of strain hardening and thermal softening may eventually deliver a net softening. Thus, although (1.1)-(1.3) is a model in the framework of isothermal mechanical theories, thermal effects are implicitly taken into account through the hypothesis of strain softening. One of our goals is to reveal similarities in the structure and predictions of (1.1)-(1.3) as compared to related models incorporating thermal effects that have been studied recently in the mathematical literature $[\mathbf{7}$, $17,19,2,1]$.

We emphasize that the spirit of this study is not to recover solutions of (1.7)-(1.4) as $n \rightarrow 0$ limits of solutions of (1.1)-(1.4). Rather, the rationale here is the converse. Because of the inherent instability induced by strain softening, it has been postulated that higher order effects, such as strain-rate dependence, play an important role and cannot be ignored (cf. $[\mathbf{1 1}, \mathbf{2 1}])$. Apart from some previous investigations using (1.3) for $n=1[\mathbf{1 8}, \mathbf{2}]$, other types of rate dependent constitutive relations have been used to analyze shear bands (e.g., Wu and Freund $[\mathbf{2 1}]$ ), as well as strain-gradient dependent constitutive laws (e.g., Coleman and Hodgdon [5]). There is a very extensive mechanics literature on the subject and the reader is referred to $[\mathbf{2 1}, \mathbf{1 1}, \mathbf{2 0}, \mathbf{1 5}]$ and references therein.

From an analysis point of view, equations (1.1)-(1.3) give rise to a coupled system consisting of a parabolic equation in $v$ coupled through the diffusion coefficient with (1.2) (cf. (3.1)). As the material is being sheared, under the effect of (1.9), the diffusion coefficient is decreasing. It is conceivable that, if the decrease is too rapid and/or nonuniform in the space variable, the diffusion may not be able to stabilize the process. 
To analyze this competition, it is helpful to recast (1.1)-(1.3) into an equivalent formulation of a reaction-diffusion system (cf. (3.4)-(3.5)).

In Section 2, we pursue an existence theory of classical solutions for a coupled system of partial differential equations (cf. (2.1)-(2.3)) that includes (1.1)-(1.3). This system also includes certain more general models in viscoelasticity with internal variables, as well as some models incorporating thermal effects that are used for the analysis of shear bands $[\mathbf{1 5}, \mathbf{1 9}]$. Motivated by the problems under consideration, the main objective is to identify a minimal set of a priori estimates sufficient for continuation of solutions. The existence theory is done in Schauder spaces, and the main ingredient is an application of the Leray-Schauder fixed point theorem. The results are summarized in Theorems 2.4 and 2.5. For existence theories of weak solutions in structurally related systems, the reader is referred to Charalambakis and Murat [3] and Nohel et al. [12].

In Section 3, we take up the problem $(\mathcal{P})_{S}$ consisting of (1.1)-(1.6) with stress boundary conditions $(1.9)_{S}$. Using the results of Section 2 together with the special structure of the system, it is shown in Theorem 3.2 that solutions of $(\mathcal{P})_{S}$ are globally defined if and only if the integral $\int_{1}^{\infty} \tau(\xi)^{1 / n} d \xi$ diverges. Moreover, the evolution of solutions of $(\mathcal{P})_{S}$ is studied under various assumptions for the constitutive function $\tau(u)$. Below, we summarize the outcome of the analysis for the special case of a power law,

$$
\sigma=\frac{1}{u^{m}} v_{x}^{n}
$$

with parameters $m, n$ positive. The parameter region is decomposed into three distinct subregions $0<m / n<1 / 2,1 / 2 \leq m / n \leq 1$ and $m / n>1$, across which the response changes drastically:

(i) In the region $0<m / n<1 / 2$, solutions of $(\mathcal{P})_{S}$ are globally defined and, as $t \rightarrow \infty$, the shear stress $\sigma(x, t)$ is attracted to the constant state $\sigma \equiv 1$ while $u(x, t)$ behaves asymptotically as a function of time.

(ii) In the region $1 / 2 \leq m / n \leq 1$, the constant state $\sigma \equiv 1$ loses its stability, and nonuniformities in the strain persist for all times.

(iii) Finally, in the region $m / n>1, u(x, t)$ becomes infinite in finite time. 
For $m / n>1 / 2$, we exhibit initial data for which the corresponding $u(x, t)$ develops nonuniformities around $x=0$ and $x=1$ and looks like two shear bands located at the boundaries. The analysis of Section 3 is effected by means of comparison principles for (3.4) and energy estimates for $(\mathcal{P})_{S}$.

For a power law (1.10) the system (1.1)-(1.2) is invariant under a family of scaling transformations. In Section 4 we take up the problem $(\mathcal{P})_{V}$ consisting of (1.1)-(1.2), (1.10) and velocity boundary conditions $(1.9)_{V}$ and introduce a change of variables motivated by the scaling property. The resulting system (4.20)-(4.22) admits positively invariant rectangles of arbitrary size. Using this observation, together with energy estimates for $(\mathcal{P})_{V}$, it is shown in Theorem 4.1 that, if $m<\min \{n, 1\}$, every solution of $(\mathcal{P})_{V}$ converges to the uniform shearing solution (1.8), as $t \rightarrow \infty$.

2. Existence theory and regularizing effect for a coupled system. We consider the initial-boundary value problem consisting of the system of quasilinear partial differential equations

$$
\begin{gathered}
\partial_{t} v=\partial_{x} w \\
\partial_{t} u=f\left(x, u, v_{x}\right)
\end{gathered}
$$

for $(x, t) \in Q_{T}:=(0,1) \times(0, T], T>0$, where

$$
w=\varphi\left(x, u, v_{x}\right),
$$

with boundary conditions

$$
v(0, t)=v(1, t)=0, \quad 0<t \leq T,
$$

or

$$
w(0, t)=w(1, t)=0, \quad 0<t \leq T,
$$

and initial conditions

$$
v(x, 0)=v_{0}(x), u(x, 0)=u_{0}(x), \quad 0 \leq x \leq 1 ;
$$

as a consequence of (2.3) and (2.5),

$$
w(x, 0)=w_{0}(x):=\varphi\left(x, u_{0}(x), v_{0 x}(x)\right) .
$$


The functions $v(x, t), w(x, t)$ are real valued, while $u(x, t)$ stands for an $\mathbf{R}^{N}$-valued function, all defined on $\bar{Q}_{T}=[0,1] \times[0, T]$. The given functions $f(x, p, q):[0,1] \times \mathbf{R}^{N} \times \mathbf{R} \rightarrow \mathbf{R}^{N}$ and $\varphi(x, p, q)$ : $[0,1] \times \mathbf{R}^{N} \times \mathbf{R} \rightarrow \mathbf{R}$ are assumed to be smooth with respect to all their arguments (the hypothesis $f$ and $\varphi$ of class $C^{2}$ suffices for all that follows). In addition, for each fixed $(x, p)$, the function $\varphi(x, p, \cdot)$ is assumed to be strictly increasing and, thus, invertible. Let $\psi(x, p, r):[0,1] \times \mathbf{R}^{N} \times \mathbf{R} \rightarrow \mathbf{R}$ be the inverse function. Inverting (2.3) yields

$$
v_{x}=\psi(x, u, w)
$$

We seek solutions $(v(x, t), u(x, t))$ of $(2.1)-(2.5)$ defined on $\bar{Q}_{T}, T>0$. Our specific goals are to identify a minimal set of a priori estimates that guarantee existence and continuation of solutions up to time $T>0$ and to study the regularizing effect that the parabolic equation $(2.1),(2.3)$ exerts on solutions.

To this end, it is expedient to state an alternative formulation of the problem. The initial-boundary value problem $(2.1)-(2.5)$ is formally equivalent to the system of reaction-diffusion equations

$$
\begin{gathered}
\partial_{t} w=a(x, u, w) \partial_{x}^{2} w+b(x, u, w) \\
\partial_{t} u=g(x, u, w)
\end{gathered}
$$

with boundary conditions

$$
w_{x}(0, t)=w_{x}(1, t)=0, \quad 0<t \leq T
$$

or

$$
w(0, t)=w(1, t)=0, \quad 0<t \leq T
$$

and initial conditions

$$
w(x, 0)=w_{0}(x), u(x, 0)=u_{0}(x), \quad 0 \leq x \leq 1,
$$

where

$$
\begin{aligned}
a(x, u, w) & =\varphi_{q}(x, u, \psi(x, u, w)) \\
b(x, u, w) & =\left(\varphi_{p} \cdot f\right)(x, u, \psi(x, u, w)) \\
g(x, u, w) & =f(x, u, \psi(x, u, w)) .
\end{aligned}
$$


Indeed, given any sufficiently smooth solution $(v(x, t), u(x, t))$ of $(2.1)-$ $(2.5)$, the pair $(w(x, t), u(x, t))$ satisfies $(2.8)-(2.11)$ as follows: Differentiating (2.3) with respect to $t$ and using (2.1), (2.2), (2.7) and (2.12), leads to $(2.8) ;(2.2)$ and $(2.7)$ yield (2.9); the rest are clear. Conversely, if $(w(x, t), u(x, t))$ is a classical solution of $(2.8)-(2.12)$, define a function $v(x, t)$ on $\bar{Q}_{T}$ such that

$$
\begin{aligned}
& v_{x}=\psi(x, u, w) \\
& v_{t}=w_{x}
\end{aligned}
$$

and $v(x, 0)=v_{0}(x), 0 \leq x \leq 1$. Since $\psi(x, p, \cdot)$ is the inverse function of $\varphi(x, p, \cdot)$, the list of relations

$$
\begin{gathered}
\varphi(x, p, \psi(x, p, r))=r \\
\varphi_{q}(x, p, \psi(x, p, r)) \psi_{r}(x, p, r)=1 \\
\varphi_{p}(x, p, \psi(x, p, r))+\varphi_{q}(x, p, \psi(x, p, r)) \psi_{p}(x, p, r)=0
\end{gathered}
$$

holds, and the compatibility of (2.13) amounts to (2.8) via (2.9) and (2.12). Moreover, $(v(x, t), u(x, t))$ satisfies (2.1)-(2.5).

Our strategy is to first prove an existence theorem for classical solutions of (2.8)-(2.11), in Schauder spaces, using the Leray-Schauder fixed point theorem [10]. This, in turn, yields an existence theorem for the equivalent system (2.1)-(2.5) provided the initial data are sufficiently smooth. The smoothness assumptions are then relaxed by means of density arguments. In the sequel $|\cdot|$ will stand for both the absolute value and the Euclidean norm in $\mathbf{R}^{N}$. Also, $\|\cdot\|_{\beta, \beta / 2}\left(|\cdot|_{\alpha}\right)$ will denote the usual Schauder norms (cf. $[\mathbf{8}, \mathbf{9}])$ in $C^{\beta, \beta / 2}\left(\bar{Q}_{T}\right)\left(C^{\alpha}[0,1]\right)$ or $\left[C^{\beta, \beta / 2}\left(\bar{Q}_{T}\right)\right]^{N}\left(\left[C^{\alpha}[0,1]\right]^{N}\right)$. The meaning of these symbols will be apparent from the context.

The possibility that (2.1)-(2.5) (or (2.8)-(2.11)) admit globally defined solutions for general nonlinear functions can be ruled out by considering special cases when the system decouples. To ensure global solvability, one could place certain growth restrictions on the functions $f$ and $\varphi$ (or $a, b$ and $g$ ). Rather than doing this, we assume that solutions of (2.1)-(2.5) or (2.8)-(2.11) satisfy certain a priori estimates, namely,

ASSUMPTION. For fixed $T>0$, there are positive constants $\mu$ and $M$, depending on norms of the initial data and $T$, such that any classical 
solution $(v(x, t), u(x, t))$ of $(2.1)-(2.5)$ on $\bar{Q}_{T}$ satisfies

$$
|w(x, t)| \leq M, \quad|u(x, t)| \leq M
$$

and

$$
\varphi_{p}\left(x, u(x, t) v_{x}(x, t)\right) \geq \mu>0
$$

for $(x, t) \in \bar{Q}_{T} ;$ correspondingly, if $(w(x, t), u(x, t))$ is a classical solution of (2.8)-(2.11) on $\bar{Q}_{T}$, then (2.15) and

$$
a(x, u(x, t), w(x, t)) \geq \mu>0
$$

hold for $(x, t) \in \bar{Q}_{T}$.

The objective is to reveal (2.15)-(2.17) as a "minimal" set of a priori estimates sufficient for continuation of solutions in some appropriate function classes. Although uniform parabolicity, embodied in (2.16) or (2.17), is not, in general, necessary for well-posedness, in light of the phenomena under consideration and for technical simplicity, solutions will be continued up to the first time that uniform parabolicity fails. For the models at hand, (2.15)-(2.17) are established in Sections 3 and 4. Finally, it is shown in Lemma 2.3 that, under natural restrictions on the initial data, (2.15)-(2.17) always hold provided $T$ is sufficiently small.

The first goal is to prove an existence theorem for (2.8)-(2.11). For this, the initial data are taken smooth, i.e.,

$$
w_{0}(x) \in C^{2+\alpha}[0,1], \quad u_{0}(x) \in\left[C^{\alpha}[0,1]\right]^{N},
$$

for some $0<\alpha<1$ and compatible with the boundary conditions

$$
w_{0 x}(i)=0, \quad i=0,1
$$

in case $(2.10)_{V}$ applies, or

$$
(2.19)_{S} w_{0}(i)=0, \quad a\left(i, u_{0}(i), 0\right) w_{0 x x}(i)+b\left(i, u_{0}(i), 0\right)=0, \quad i=0,1,
$$

in case $(2.10)_{S}$ applies. We prove 
THEOREM 2.1. Let $\left(w_{0}(x), u_{0}(x)\right)$ satisfy (2.18), (2.19) and assume that the a priori estimates (2.15) and (2.17) hold for some $T>0$, with $M$ and $\mu$ positive constants depending at most on $\left\|w_{0}\right\|_{2+\alpha},\left\|u_{0}\right\|_{\alpha}$ and $T$. There exists a unique solution $(w(x, t), u(x, t))$ of $(2.8)-(2.11)$ on $\bar{Q}_{T}$ such that $w, w_{t}, w_{x}, w_{x x}$ are in $C^{\alpha, \alpha / 2}\left(\bar{Q}_{T}\right)$ and $u, u_{t}$ are in $\left[C^{\alpha, \alpha / 2}\left(\bar{Q}_{T}\right)\right]^{N}$.

PROOF. The proof of uniqueness is lengthy, but routine, and it is omitted.

In view of (2.15) and (2.17), the triplet $(x, u(x, t), w(x, t))$ takes values in the set $E=\left\{(x, u, w) \in[0,1] \times \mathbf{R}^{N} \times \mathbf{R}:|u| \leq M\right.$, $|w| \leq M, a(x, u, w) \geq \mu\}$. By modifying, if necessary, the functions $a, b$ and $g$ outside some open set containing $E$, it is assumed for the existence part of the proof that all the functions involved are bounded, globally Lipschitz, and, wherever appropriate, with globally Lipschitz derivatives. Moreover,

$$
a(x, p, r) \geq \frac{\mu}{2}>0
$$

for $(x, p, r) \in[0,1] \times \mathbf{R}^{N} \times \mathbf{R}$. All bounds and Lipschitz constants depend only on $M$ and $\mu$. For the remainder of the proof, $K$ will stand for a generic constant that can be estimated solely in terms of $M, \mu$ and $T$.

We work with the boundary conditions $(2.10)_{S}$; the boundary conditions $(2.10)_{V}$ are treated similarly. Let $\mathcal{B}$ denote the Banach space

$$
\mathcal{B}=\left\{w(x, t) \in C^{\beta, \beta / 2}\left(\bar{Q}_{T}\right): w(0, t)=w(1, t)=0,0 \leq t \leq T\right\}
$$

and let $\mathcal{C}$ stand for the closed subset of $\left[C^{\beta, \beta / 2}\left(\bar{Q}_{T}\right)\right]^{N}$ :

$$
\mathcal{C}=\left\{u(x, t) \in\left[C^{\beta, \beta / 2}\left(\bar{Q}_{T}\right)\right]^{N}: u(x, 0)=u_{0}(x), 0 \leq x \leq 1\right\} .
$$

For our purposes, $\beta=\min \{\alpha, 1 / 2\}$. Define the map $\mathcal{T}: \mathcal{B} \rightarrow \mathcal{C}$ that carries $W(x, t) \in \mathcal{B}$ to $U(x, t)$, the solution of the family of initial value problems

$$
\begin{array}{cl}
U_{t}=g(x, U, W(x, t)), & 0 \leq x \leq 1,0 \leq t \leq T, \\
U(x, 0)=u_{0}(x), & 0 \leq x \leq 1 .
\end{array}
$$


Also, for $\lambda \in[0,1]$, define a second map $\mathcal{S}:[0,1] \times \mathcal{B} \times \mathcal{C} \rightarrow \mathcal{B}$ which takes $(\lambda, W(x, t), U(x, t))$ to $w(x, t)$, the solution of the initial-boundary value problem

$$
\begin{gathered}
w_{t}=a_{\lambda}(x, U(x, t), W(x, t)) w_{x x} \\
+\lambda b(x, U(x, t), W(x, t))+(1-\lambda) F(x), \\
w(0, t)=w(1, t)=0, \quad 0 \leq t \leq T \\
w(x, 0)=w_{0}(x), \quad 0 \leq x \leq 1,
\end{gathered}
$$

where $a_{\lambda}(x, p, r):=\lambda a(x, p, r)+(1-\lambda)(\mu / 2) \geq(\mu / 2)$, by $(2.20)$, and $F(x):=-(\mu / 2) w_{0 x x}(x)$.

Given the maps $\mathcal{T}$ and $\mathcal{S}$, construct the composite map $P:[0,1] \times \mathcal{B} \rightarrow$ $\mathcal{B}$ which carries $\lambda \in[0,1], W \in \mathcal{B}$ to

$$
w=P(\lambda, W):=\mathcal{S}(\lambda, W, \mathcal{T}(W)) .
$$

Observe that if $w(x, t) \in \mathcal{B}$ is a fixed point of $P(1, \cdot)$ and $u(x, t)$ the corresponding solution of $(2.23)$, then $(w(x, t), u(x, t))$ satisfies (2.8)-(2.11) on $\bar{Q}_{T}$. Our objective is to demonstrate that the map $P$ fulfills the hypotheses of the Leray-Schauder fixed point theorem (for a formulation, see [10], also [6]). To this end, certain properties of the maps $\mathcal{T}$ and $\mathcal{S}$ are recorded below.

First, consider the map $\mathcal{T}: \mathcal{B} \rightarrow \mathcal{C}$. Since $g$ is bounded and globally Lipschitz, the standard theory of ordinary differential equations implies that, given any $W(x, t) \in \mathcal{B}$, there is a unique solution $U(x, t)$ of $(2.23)$ defined on $[0,1] \times[0, T]$ and such that

$$
|U(x, t)|+\left|U_{t}(x, t)\right| \leq K_{1} .
$$

Moreover, by first integrating $(2.23)_{1}$ for two distinct points $x_{1}, x_{2}$ in $[0,1]$ over $[0, t], 0<t \leq T$, and then, estimating the difference using Gronwall's inequality, we deduce, with the help of (2.26),

$$
\|U\|_{\beta, \beta / 2} \leq K_{2}\left(\left|u_{0}\right|_{\alpha}+\|W\|_{\beta, \beta / 2}+1\right)
$$

Next, consider the map $\mathcal{S}:[0,1] \times \mathcal{B} \times \mathcal{C} \rightarrow \mathcal{B}$. The classical Schauder theory for parabolic equations $[\mathbf{8}, \mathbf{9}]$ implies that, given any triplet 
$(\lambda, W, U) \in[0,1] \times \mathcal{B} \times \mathcal{C}$, there is a unique solution $w(x, t)$ of $(2.24)$ on $\bar{Q}_{T}$ belonging to $C^{2+\beta, 1+\beta / 2}\left(\bar{Q}_{T}\right)$ and satisfying

$$
\|w\|_{2+\beta, \beta / 2} \leq \Lambda_{1}\left(\left|w_{0}\right|_{2+\alpha}+\|W\|_{\beta, \beta / 2}+\|U\|_{\beta, \beta / 2}+1\right) .
$$

The constant $\Lambda_{1}$ can be estimated solely in terms of $\|W\|_{\beta, \beta / 2}$, $\|U\|_{\beta, \beta / 2}, \mu, M$ and $T$.

Finally, consider the map $P:[0,1] \times \mathcal{B} \rightarrow \mathcal{B} . \quad P$ is well defined by (2.25). Also:

(i) For any fixed $\lambda \in[0,1], P(\lambda, \cdot): \mathcal{B} \rightarrow \mathcal{B}$ is compact and continuous. Since the injection $C^{2+\beta, 1+\beta / 2}\left(\bar{Q}_{T}\right) \rightarrow C^{\beta, \beta / 2}\left(\bar{Q}_{T}\right)$ is compact, (2.27) and (2.28) imply that $P(\lambda, \cdot)$ is a compact map. Let $\left\{W_{n}\right\}$ be a convergent sequence in $\mathcal{B}, W_{n} \rightarrow W$ in $C^{\beta, \beta / 2}\left(\bar{Q}_{T}\right)$; consider $w_{n}=P\left(\lambda, W_{n}\right)$. Since $P(\lambda, \cdot)$ is a compact map, along a subsequence, $w_{n} \rightarrow w$ in $C^{\beta, \beta / 2}$ (in fact, in $C^{2+\beta^{\prime}, 1+\beta^{\prime} / 2}$, for any $\beta^{\prime}<\beta$ ). One easily shows that $w=P(\lambda, W)$. Since $P(\lambda, \cdot)$ is single-valued, $w_{n} \rightarrow w$ along the whole sequence and $P(\lambda, \cdot)$ is continuous.

(ii) For any bounded subset $\mathcal{K}$ of $\mathcal{B}$, the family of maps $P(\cdot, W)$ : $[0,1] \rightarrow \mathcal{B}, W \in \mathcal{K}$, is uniformly equicontinuous. Let $\mathcal{K}$ be a bounded subset of $\mathcal{B}$. Fix $W \in \mathcal{K}$. For $U=\mathcal{T}(W)$ and $\lambda, \rho$ in $[0,1]$, let $w_{\lambda}$, $w_{\rho}$ be the respective solutions of (2.24). Note that $w_{\lambda}=P(\lambda, W)$, $w_{\rho}=P(\rho, W)$. The difference $w_{\lambda}-w_{\rho}$ satisfies the parabolic equation

$$
\begin{aligned}
\left(w_{\lambda}-w_{\rho}\right)_{t}= & a_{\lambda}(x, U(x, t), W(x, t))\left(w_{\lambda}-w_{\rho}\right)_{x x} \\
& +(\lambda-\rho)\left[a(x, U(x, t), W(x, t)) w_{\rho x x}\right. \\
& \left.+b(x, U(x, t), W(x, t))-\left(\frac{\mu}{2} w_{\rho x x}+F(x)\right)\right],
\end{aligned}
$$

with boundary conditions $(2.24)_{2}$ and initial condition $\left(w_{\lambda}-w_{\rho}\right)(x, 0)=$ 0 . The Schauder estimates imply that

$$
\begin{array}{r}
\left\|w_{\lambda}-w_{\rho}\right\|_{2+\beta, 1+\beta / 2} \\
\leq \Lambda_{2}|\lambda-\rho|\left\{\left\|w_{\rho}\right\|_{2+\beta, 1+\beta / 2}+\|W\|_{\beta, \beta / 2}\right. \\
\left.+\|U\|_{\beta, \beta / 2}+\left|w_{0}\right|_{2+\alpha}+1\right\}
\end{array}
$$

with $\Lambda_{2}$ a constant as in (2.28). Combining (2.27), (2.28), and (2.30), we arrive at

$$
\left\|w_{\lambda}-w_{\rho}\right\|_{2+\beta, 1+\beta / 2} \leq \Lambda_{3}|\lambda-\rho|
$$


with $\Lambda_{3}$ depending only on $\mathcal{K}$, the initial data, $\mu, M$ and $T$. Thus, $P(\cdot, W), W \in \mathcal{K}$ is a uniformly equicontinuous family of maps.

(iii) $P(0, \cdot)$ has precisely one fixed point in $\mathcal{B}$. For $\lambda=0,(2.24)_{1}$ becomes the heat equation, (2.23) and (2.24) decouple, and (2.24) has a unique solution in $\mathcal{B}$.

(iv) Any fixed point in $\mathcal{B}$ of $P(\lambda, \cdot), 0 \leq \lambda \leq 1$, is contained in some bounded subset $\mathcal{K}$ of $\mathcal{B}$. Let $w \in \mathcal{B}$ be a fixed point of $P(\lambda, \cdot)$. Set $u=\mathcal{T}(w)$. Then (2.27) implies $u \in\left[C^{\beta, \beta / 2}\left(\bar{Q}_{T}\right)\right]^{N}$, and (2.26) now reads

$$
|u(x, t)|+\left|u_{t}(x, t)\right| \leq K_{3}, \quad(x, t) \in \bar{Q}_{T} .
$$

By $(2.27)$ and $(2.28), w \in C^{2+\beta, 1+\beta / 2}\left(\bar{Q}_{T}\right)$ and satisfies

$$
w_{t}=a_{\lambda}(x, u, w) w_{x x}+\lambda b(x, u, w)+(1-\lambda) F(x)
$$

on $\bar{Q}_{T}$ with boundary and initial conditions as in (2.24). Since $b$ is bounded, the maximum principle yields

$$
|w(x, t)| \leq \sup _{0 \leq x \leq 1}\left|w_{0}(x)\right|+K_{4}+(1-\lambda) T \sup _{0 \leq x \leq 1}|F(x)|, \quad(x, t) \in \bar{Q}_{T} .
$$

Next, we multiply $(2.33)$ by $w_{t} / a_{\lambda}$, integrate by parts over $[0,1] \times[0, t]$, and use Schwarz's inequality to deduce

$$
\begin{aligned}
& \int_{0}^{t} \int_{0}^{1} w_{t}^{2} d x d \tau+\int_{0}^{1} w_{x}^{2}(x, t) d x \\
& \quad \leq K_{5}\left[1+\int_{0}^{1} w_{0 x}^{2}(x) d x+(1-\lambda)^{2} \sup _{0 \leq x \leq 1}|F(x)|^{2}\right]=: C^{2} .
\end{aligned}
$$

Using (2.35), we obtain

$$
|w(x, t)-w(y, t)| \leq C|x-y|^{1 / 2}
$$

Also, for fixed $\delta>0$, the calculus inequality

$$
\begin{aligned}
& 2 \delta|w(x, t)-w(x, \tau)| \leq \int_{x-\delta}^{x+\delta}|w(x, t)-w(y, t)| d y \\
& \quad+\int_{x-\delta}^{x+\delta}\left|\int_{\tau}^{t} w_{s}(y, s) d s\right| d y+\int_{x-\delta}^{x+\delta}|w(y, \tau)-w(x, \tau)| d y
\end{aligned}
$$


holds. We estimate (2.37), using (2.36) and (2.35), and in the resulting inequality we set $\delta=|t-\tau|^{1 / 2}$ to arrive at

$$
|w(x, t)-w(x, \tau)| \leq 3 C|t-\tau|^{1 / 4} .
$$

On account of $(2.34),(2.36)$ and (2.38), any fixed point $w \in \mathcal{B}$ is contained in a bounded set of $C^{1 / 2,1 / 4}\left(\bar{Q}_{T}\right)$, and, since $\beta=\min \{\alpha, 1 / 2\}$, also in a bounded set of $\mathcal{B}$.

The map $P$ fulfills the hypotheses of the Leray-Schauder fixed point theorem. Thus, the map $P(1, \cdot)$ has a fixed point in $\mathcal{B}$. If $w \in \mathcal{B}$ is such a fixed point and $u=\mathcal{T}(w)$, then $(w(x, t), u(x, t))$ is a classical solution of $(2.8)-(2.11)$ on $[0,1] \times[0, T]$. $\square$

We collect in Lemma 2.2 certain a priori estimates for solutions of (2.8)-(2.11) that serve as a starting point to develop an existence theory for the system (2.1)-(2.5). Estimates (2.41) capture the regularizing effect of the parabolic equation (2.8).

LEMMA 2.2. Let $(w(x, t), u(x, t))$ be a classical solution of $(2.8)-(2.11)$ on $\bar{Q}_{T}$ satisfying (2.15) and (2.17). Then

$$
\begin{gathered}
\|w\|_{\frac{1}{2}, \frac{1}{4}} \leq C_{1}\left(1+\int_{0}^{1} w_{0 x}^{2}(x) d x\right), \\
\|u\|_{\beta, \beta / 2} \leq C_{2}\left(1+\int_{0}^{1} w_{0 x}^{2}(x) d x+\left|u_{0}\right|_{\alpha}\right),
\end{gathered}
$$

where $\beta=\min \{\alpha, 1 / 2\}$. Moreover, for any $x, y \in[0,1], s, \tau \in[t, T]$ with $t>0$,

$$
\begin{aligned}
& |w(x, \tau)-w(y, \tau)| \leq \frac{C_{3}}{\sqrt{t}}|x-y|^{1 / 2} \\
& |w(x, \tau)-w(x, s)| \leq \frac{3 C_{3}}{\sqrt{t}}|\tau-s|^{1 / 4} .
\end{aligned}
$$

The constants $C_{1}, C_{2}$ and $C_{3}$ above depend only on $\mu, M$ and $T$.

ProOF. Estimate (2.39) is a direct consequence of (2.34) and (2.35) with $\lambda=1$, together with (2.36) and (2.38); (2.40) follows by combining (2.27) with (2.39). 
To show (2.41), first multiply (2.8) by $w / a(x, u, w)$ and write the resulting identity in the form

$$
\begin{gathered}
\partial_{t} \int_{w_{0}(x)}^{w} \frac{\xi}{a(x, u, \xi)} d \xi+w_{x}^{2} \\
\quad=\left(w w_{x}\right)_{x}+\frac{w b(x, u, w)}{a(x, u, w)}-u_{t} \cdot \int_{w_{0}(x)}^{w} \frac{\xi a_{u}(x, u, \xi)}{a^{2}(x, u, \xi)} d \xi .
\end{gathered}
$$

In view of (2.17), we may assume that (2.20) holds. Integrating (2.42) over $[0,1] \times[0, t], 0<t \leq T$ and using (2.9), (2.10), (2.15) and (2.20), we obtain

$$
\int_{0}^{t} \int_{0}^{1} w_{x}^{2} d x d \tau \leq K_{1}
$$

Next, multiply $(2.8)$ by $\left(t w_{t}\right) / a(x, u, w)$ and integrate by parts over $[0,1] \times[0, t]$; by estimating the resulting identity via $(2.15),(2.20)$, Schwarz's inequality and (2.43), we conclude that

$$
\int_{0}^{t} \int_{0}^{1} \tau w_{t}^{2}(x, \tau) d x d \tau+t \int_{0}^{1} w_{x}^{2}(x, t) d x \leq K_{2} .
$$

$K_{1}$ and $K_{2}$ depend only on $\mu, M$ and $T$. The derivation of (2.41) from (2.44) is similar to the derivation of (2.36) and (2.38) from (2.35); in (2.41), $C_{3}=\sqrt{K_{2}}$. $\square$

Lemma 2.3 guarantees that the a priori estimates (2.15) and (2.17) required in the hypotheses of Theorem 2.1 are always valid for $T$ sufficiently small.

LEMMA 2.3. Let $(w(x, t), u(x, t))$ be a classical solution of $(2.8)-(2.11)$ defined on $[0,1] \times\left[0, T^{*}\right)$, for some $T^{*}>0$.

(a) Suppose that

$$
m_{1}:=\min _{0 \leq x \leq 1} a\left(x, u_{0}(x), w_{0}(x)\right)>0 .
$$

Then, given any positive constants $\mu, M$ with $\mu<m_{1}$, there is a $T<T^{*}$ depending on $\mu, M$ and the $L^{\infty}$-norm of $w_{0 x x}$ such that

$$
\left|w(x, t)-w_{0}(x)\right| \leq M,
$$




$$
\left|u(x, t)-u_{0}(x)\right| \leq M
$$

and (2.17) hold for $(x, t) \in \bar{Q}_{T}$.

(b) Suppose that, in addition,

$$
m_{2}:=\min _{\substack{0 \leq x \leq 1 \\ w_{0} \leq \leq \leq w_{0_{+}}}} a\left(x, u_{0}(x), r\right)>0,
$$

where $w_{0_{-}}=\inf _{0 \leq x \leq 1} w_{0}(x), w_{0_{+}}=\sup _{0 \leq x \leq 1} w_{0}(x)$. Then, given any $\mu, M, M_{-}, M_{+}$with $0<\mu<m_{2}, M>0, M_{-}<w_{0_{-}} \leq w_{0_{+}}<M_{+}$, there is a $T<T^{*}$ depending solely on $\mu, M, M_{-}$and $M_{+}$such that (2.17), (2.47) and

$$
M_{-} \leq w(x, t) \leq M_{+}
$$

hold for $(x, t) \in \bar{Q}_{T}$.

Proof. Let $W=w-w_{0}, U=u-u_{0}$. Then, $(W(x, t), U(x, t))$ satisfy, on $[0,1] \times\left[0, T^{*}\right)$, the differential equations

$$
\begin{aligned}
W_{t}-A(x, U, W) W_{x x} & =A(x, U, W) w_{0 x x}(x)+B(x, U, W) \\
U_{t} & =G(x, U, W)
\end{aligned}
$$

with boundary conditions (2.10) and initial conditions $W(x, 0)=0$, $U(x, 0)=0$, for $0 \leq x \leq 1$; the functions $A, B$ and $G$ relate to $a, b$ and $g$ through formulas of the format

$$
A(x, U, W)=a\left(x, u_{0}(x)+U, w_{0}(x)+W\right) .
$$

First, consider part (a). Since $a, w_{0}$ and $u_{0}$ are continuous, (2.45) and (2.52) imply that there is a $\rho>0$ so that if $0 \leq x \leq 1,|U| \leq \rho$ and $|W| \leq \rho$, then $A(x, U, W) \geq \mu>0$. Set $k=\min \{\rho, M\}$. To complete the proof of part (a), it suffices to show that there is a $T<T^{*}$ such that, for $(x, t) \in \bar{Q}_{T}$, the triplet

$$
\begin{aligned}
(x, U(x, t), W(x, t)) \in E_{1}:= \\
\left\{(x, U, W) \in[0,1] \times R^{N} \times R:|W| \leq k,|U| \leq k\right\} .
\end{aligned}
$$

Then (2.17), (2.46) and (2.47) follow from (2.52) and (2.53). 
Clearly, (2.53) holds on $\bar{Q}_{\tau}$ for some $\tau$ sufficiently small. Moreover, so long as (2.53) holds, the maximum principle for the parabolic equation (2.50) gives the bound

$$
|W(x, t)| \leq F_{1} t
$$

where $F_{1}=\sup _{E_{1}}\left|A(x, U, W) w_{0 x x}(x)+B(x, U, W)\right|$. Also, if $G_{1}=$ $\sup _{E_{1}}|G(x, U, W)|$, then (2.51), together with Gronwall's inequality, yields

$$
|U(x, t)| \leq G_{1} t .
$$

Finally, (2.54) and (2.55) imply that (2.53) holds on $\bar{Q}_{T}$, for any $0<T<T^{*}$ with $T \leq \min \left\{k / F_{1}, k / G_{1}\right\}$. Since $F_{1}$ depends on the $L^{\infty}$-norm of $w_{0 x x}$, the resulting $T$ will exhibit the same dependence. Under the stronger hypothesis (2.48), this dependence can be avoided.

Consider now part (b). By virtue of (2.48) and the continuity of $a$ and $u_{0}$, there are $\rho, \rho_{-}, \rho_{+}$, with $\rho>0$ and $\rho_{-}<w_{0_{-}} \leq w_{0_{+}}<\rho_{+}$such that $0 \leq x \leq 1,|U| \leq \rho$, and $\rho_{-} \leq w \leq \rho_{+}$imply $a\left(x, u_{0}(x)+U, w\right) \geq \mu>0$. It now suffices to show that there is a $T<T^{*}$ such that, for $(x, t) \in \bar{Q}_{T}$, the triplet

$$
(x, U(x, t), w(x, t)) \in E_{2}:=[0,1] \times\left\{U \in \mathbf{R}^{N}:|U| \leq k\right\} \times\left[k_{-}, k_{+}\right] ;
$$

here, $k=\min \{\rho, M\}, k_{-}=\max \left\{\rho_{-}, M_{-}\right\}, k_{+}=\min \left\{\rho_{+}, M_{+}\right\}$and $k_{-}<w_{0_{-}} \leq w_{0_{+}}<k_{+}$.

Let $B_{ \pm}=\sup \max _{E_{2}}\left\{0, \pm b\left(x, u_{0}(x)+U, w\right)\right\}$, and consider the comparison functions $W_{ \pm}(x, t)= \pm B_{ \pm} t-w_{0}(x)+w_{0 \pm}$. On account of (2.50), so long as (2.56) holds, the functions $W_{ \pm}$satisfy the differential inequalities

$$
\begin{gathered}
\partial_{t} W_{-} A(x, U, W) \partial_{x}^{2} W_{-} \leq \partial_{t} W-A(x, U, W) \partial_{x}^{2} W \\
\leq \partial_{t} W_{+}-A(x, U, W) \partial_{x}^{2} W_{+} \\
W_{-}(x, 0) \leq W(x, 0)=0 \leq W_{+}(x, 0)
\end{gathered}
$$

and, by (2.19), corresponding inequalities at the boundaries. Using comparison principles for parabolic equations, we obtain

$$
-B_{-} t+w_{0-} \leq w(x, t) \leq B_{+} t+w_{0+} .
$$


Moreover, (2.51) yields

$$
|U(x, t)| \leq G_{2} t
$$

where $G_{2}=\sup _{E_{2}}\left|g\left(x, u_{0}(x)+U, w\right)\right|$. Finally, (2.58) and (2.59) imply that (2.56) holds on $\bar{Q}_{T}$ for any $0<T<T^{*}$ with $T \leq$ $\min \left\{\left(k_{+}-w_{0+}\right) / B_{+},\left(w_{0_{-}}-k_{-}\right) / B_{-}, k / G_{2}\right\}$. $\square$

Theorem 2.1, in conjunction with Lemmas 2.2 and 2.3, gives rise to the following local existence and continuation theorem for the initialboundary value problem (2.8)-(2.11) in Schauder spaces.

THEOREM 2.4. Let $w_{0}(x) \in C^{1+\alpha}[0,1], u_{0}(x) \in\left[C^{\alpha}[0,1]\right]^{N}$ satisfy the compatibility conditions $w_{0 x}(0)=w_{0 x}(1)=0$ in case $(2.10)_{V}$ applies, or $w_{0}(0)=w_{0}(1)=0$ in case $(2.10)_{S}$ applies, and suppose that $(2.48)$ holds. Then, there exists a unique classical solution $(w(x, t), u(x, t))$ of (2.8)-(2.11) defined on a maximal interval of existence $[0,1] \times\left[0, T^{*}\right)$ such that, for any $0<\tau<T<T^{*}, w$ is in $C^{\alpha, \alpha / 2}\left(\bar{Q}_{T}\right), u, u_{t}$ are in $\left[C^{\alpha, \alpha / 2}\left(\bar{Q}_{T}\right)\right]^{N}$ and $w_{t}, w_{x}, w_{x x}$ are in $C^{\alpha, \alpha / 2}([0,1] \times[\tau, T])$. In case $T^{*}<\infty$, as $t \uparrow T^{*}$,

$$
\limsup _{t \uparrow T^{*}} \sup _{0 \leq x \leq 1}(|u(x, t)|+|w(x, t)|)=\infty
$$

and/or

$$
\liminf _{t \uparrow T^{*}} \inf _{0 \leq x \leq 1} a(x, u(x, t), w(x, t))=0 .
$$

Furthermore, if $w_{0}(x) \in C^{2+\alpha}[0,1]$ and the compatibility conditions (2.19) hold, then $w_{t}, w_{x}, w_{x x}$ are in $C^{\alpha, \alpha / 2}\left(\bar{Q}_{T}\right)$ for any $T<T^{*}$. If, in addition, $u_{0}(x) \in\left[C^{1+\alpha}[0,1]\right]^{N}$, then $u_{x}, u_{x t}$ are in $\left[C^{\alpha, \alpha / 2}\left(\bar{Q}_{T}\right)\right]^{N}$ for any $T<T^{*}$.

PROOF. We work with the boundary conditions $(2.10)_{S}$; the case of $(2.10)_{V}$ is treated similarly.

Let $w_{0}(x) \in C^{1+\alpha}[0,1], u_{0}(x) \in\left[C^{\alpha}[0,1]\right]^{N}$ be given, satisfying the compatibility conditions $w_{0}(0)=w_{0}(1)=0$ as well as (2.48). 
We proceed to establish a local existence theorem for (2.8)-(2.11). First, construct approximating sequences $\left\{w_{0 n}\right\}$ and $\left\{u_{0 n}\right\}$ such that $w_{0 n}(x)$ and $u_{0 n}(x)$ are $C^{\infty}$-functions on $[0,1]$ satisfying $(2.19)_{S}$, and, as $n \rightarrow \infty$,

$$
\begin{array}{llrl}
w_{0 n} \rightarrow w_{0} & \text { in } C^{1}[0,1], & \left|w_{0 n}\right|_{1+\alpha} \leq K\left|w_{0}\right|_{1+\alpha} \\
u_{0 n} \rightarrow u_{0} & \text { in }[C[0,1]]^{N}, & \left|u_{0 n}\right|_{\alpha} \leq K\left|u_{0}\right|_{\alpha},
\end{array}
$$

with $K$ a fixed positive constant (for details of such a construction, see $[\mathbf{1 9}])$.

Consider the problem (2.8)-(2.11) with initial data $\left(w_{0 n}(x), u_{0 n}(x)\right)$. Referring to part (b) of Lemma 2.3, let

$m_{n}=\min \left\{a\left(x, u_{0 n}(x), r\right): 0 \leq x \leq 1, \inf _{0 \leq x \leq 1} w_{0 n}(x) \leq r \leq \sup _{0 \leq x \leq 1} w_{0 n}(x)\right\}$.

Since $\left(w_{0}(x), u_{0}(x)\right)$ satisfy $(2.48)$ and $u_{0}(x)$ is continuous, $\liminf \inf _{n \rightarrow \infty}$ $m_{n}>0$. By throwing away a finite number of terms, if needed, we may assume that $m_{0}:=\inf _{n} m_{n}>0$. Fix $\mu, M, M_{-}$and $M_{+}$ such that $M_{-}<\inf _{n} \inf _{0 \leq x \leq 1} w_{0 n}(x) \leq \sup _{n} \sup _{0 \leq x \leq 1} w_{0 n}(x)<$ $M_{+}, M>0,0<\mu<m_{0}$. Theorem 2.1, in conjunction with Lemma 2.3, implies that, for each $n=1,2, \ldots$, there is a classical solution $\left(w_{n}(x, t), u_{n}(x, t)\right)$ of $(2.8)-(2.11)$ defined on $[0,1] \times\left[0, T_{n}\right]$, with smoothness as in Theorem 2.1, and corresponding to the initial data $\left(w_{0 n}(x), u_{0 n}(x)\right)$. Moreover, $T_{0}:=\inf _{n} T_{n}>0$, and, on the domain $\bar{Q}_{T_{0}}=[0,1] \times\left[0, T_{0}\right]$, the functions $\left(w_{n}(x, t), u_{n}(x, t)\right)$ satisfy the uniform bounds

$$
\left|u_{n}(x, t)-u_{0 n}(x)\right| \leq M, \quad M_{-} \leq w_{n}(x, t) \leq M_{+}
$$

and

$$
a\left(x, u_{n}(x, t), w_{n}(x, t)\right) \geq \mu>0 .
$$

Using (2.62), (2.63), (2.65) and (2.66), relations (2.39) and (2.40) in Lemma 2.2, together with (2.9), imply that, on $\bar{Q}_{T_{0}}$,

$$
\left\|w_{n}\right\|_{\beta, \beta / 2} \leq K, \quad\left\|u_{n}\right\|_{\beta, \beta / 2}+\left\|\partial_{t} u_{n}\right\|_{\beta, \beta / 2} \leq K,
$$

where $\beta=\min \{\alpha, 1 / 2\}$ and $K$ is a constant independent of $n$. 
Since the injection $C^{\beta, \beta / 2}\left(\bar{Q}_{T_{0}}\right) \rightarrow C^{\beta^{\prime}, \beta^{\prime} / 2}\left(\bar{Q}_{T_{0}}\right)$ is compact for $\beta^{\prime}<\beta,(2.67)$ implies that there are subsequences $\left\{w_{n^{\prime}}\right\}$ and $\left\{u_{n^{\prime}}\right\}$, as well as functions $w(x, t)$ and $u(x, t)$, with $w \in C^{\beta, \beta / 2}\left(\bar{Q}_{T_{0}}\right)$ and $u$, $\partial_{t} u \in\left[C^{\beta, \beta / 2}\left(\bar{Q}_{T_{0}}\right)\right]^{N}$, such that

$$
\begin{gathered}
w_{n^{\prime}} \rightarrow w \quad \text { in } C^{\beta^{\prime}, \beta^{\prime} / 2}\left(\bar{Q}_{T_{0}}\right), \\
u_{n^{\prime}} \rightarrow u, \partial_{t} u_{n^{\prime}} \rightarrow \partial_{t} u \quad \text { in }\left[C^{\beta^{\prime}, \beta^{\prime} / 2}\left(\bar{Q}_{T_{0}}\right)\right]^{N} .
\end{gathered}
$$

Clearly, $(w(x, t), u(x, t))$ satisfies $(2.9),(2.10)_{S}$ and (2.11). Using (2.68) together with results on families of solutions of parabolic equations (cf. Friedman [8, Section 3.6]), it follows that $(w(x, t), u(x, t)$ is a classical solution of (2.8). The stated regularity of this solution is an outcome of the interior and boundary parabolic estimates $[\mathbf{8}$, Section 4.7]. Uniqueness follows from a lengthy but routine argument that is omitted.

If $w_{0}(x) \in C^{2+\alpha}[0,1]$ and satisfies $(2.19)_{S}$, then Theorem $2.1 \mathrm{im}-$ plies that $w_{t}, w_{x}$ and $w_{x x}$ are in $C^{\alpha, \alpha / 2}\left(\bar{Q}_{T_{0}}\right)$. Suppose that, in addition, $u_{0}(x) \in\left[C^{1+\alpha}[0,1]\right]^{N}$. Now $u(x, t)$ satisfies (2.9) with $w, w_{x} \in$ $C^{\alpha, \alpha / 2}\left(\bar{Q}_{T_{0}}\right)$. Using standard theorems on continuous dependence for ordinary differential equations, together with estimates in the spirit of the derivation of (2.27), leads to $u_{x}, u_{x t} \in\left[C^{\alpha, \alpha / 2}\left(\bar{Q}_{T_{0}}\right)\right]^{N}$.

Finally, Theorem 2.1 implies that the solution $(w(x, t), u(x, t))$ can be continued on a maximal interval of existence $[0,1] \times\left[0, T^{*}\right)$, such that either $T^{*}=\infty$, or, at least one of (2.60) or (2.61) occurs. $\square$

Next, we turn to the initial-boundary value problem (2.1)-(2.5). We assume that the initial data satisfy

$$
\begin{gathered}
v_{0}(x) \in C^{2+\alpha}[0,1], \quad u_{0}(x) \in\left[C^{1+\alpha}[0,1]\right]^{N}, \\
m_{0}=\min \left\{\varphi _ { q } \left(x, u_{0}(x), \psi\left(x, u_{0}(x), r\right): 0 \leq x \leq 1,\right.\right. \\
\left.\inf _{0 \leq x \leq 1} w_{0}(x) \leq r \leq \sup _{0 \leq x \leq 1} w_{0}(x)\right\}>0,
\end{gathered}
$$

where $w_{0}(x)$ is given by (2.6), and the compatibility conditions

$$
v_{0}(0)=v_{0}(1)=0, \quad w_{0 x}(0)=w_{0 x}(1)=0,
$$


in case $(2.4)_{V}$ applies, or

$$
w_{0}(0)=w_{0}(1)=0
$$

in case $(2.4)_{S}$ applies. We prove

THEOREM 2.5. Under the hypotheses (2.69)-(2.71), there exists a unique classical solution $(v(x, t), u(x, t))$ of $(2.1)-(2.5)$ defined on a maximal interval of existence $[0,1] \times\left[0, T^{*}\right)$ such that, for any $0<T<T^{*}, v, v_{x}, v_{t}, v_{x x}$ are in $C^{\alpha, \alpha / 2}\left(\bar{Q}_{T}\right)$ and $u, u_{t}, u_{x}, u_{x t}$ are in $\left[C^{\alpha, \alpha / 2}\left(\bar{Q}_{T}\right)\right]^{N}$. If $T^{*}<\infty$, then, as $t \uparrow T^{*}$, at least one out of (2.60)

or

$$
\liminf _{t \uparrow T^{*}} \inf _{0 \leq x \leq 1} \varphi_{q}\left(x, u(x, t), v_{x}(x, t)\right)=0
$$

occurs. Finally, $w(x, t)=\varphi\left(x, u(x, t), v_{x}(x, t)\right)$ satisfies (2.41), where $C_{3}$ only depends on $m_{0}$ and the sup-norms of $w_{0}$ and $u_{0}$.

Proof. For concreteness, we treat the boundary conditions $(2.4)_{S}$. Let $\left(v_{0}(x), u_{0}(x)\right)$ satisfying $(2.69),(2.70)$ and $(2.71)_{S}$ be given and define $w_{0}(x)$ by (2.6). Consider the problem (2.8)-(2.11) with $a, b$ and $g$ defined by (2.12). Theorem 2.4 asserts that there is a unique solution $(w(x, t), u(x, t))$ of $(2.8)-(2.11)$ defined on $[0,1] \times\left[0, T^{*}\right)$ and with regularity as stated there.

Our objective is to define $v(x, t)$ by (2.13) subject to the initial data $v(x, 0)=v_{0}(x), 0 \leq x \leq 1$. Then $(v(x, t), u(x, t))$ is a solution of $(2.1)-(2.5)$. For $v(x, t)$ to be well defined, it is at least required that, for each fixed $x \in[0,1], w_{x}(x, \cdot)$ is integrable. In view of Theorem 2.4 , to complete the proof it suffices to show that, for some $\tau$ small, $w_{x}, v_{t}, v_{x x} \in C^{\alpha, \alpha / 2}\left(\bar{Q}_{\tau}\right), u_{x}, u_{x t} \in\left[C^{\alpha, \alpha / 2}\left(\bar{Q}_{\tau}\right)\right]^{N}$ and also that (3.41) holds. This is accomplished by a density argument.

Consider approximating sequences $\left\{w_{0 n}\right\}$ and $\left\{u_{0 n}\right\}$, consisting of $C^{\infty}$-functions on $[0,1]$, with $\left\{w_{0 n}\right\}$ as in (2.62) and $\left\{u_{0 n}\right\}$ satisfying

$$
u_{0 n} \rightarrow u_{0} \text { in }\left[C^{1}[0,1]\right]^{N}, \quad\left|u_{0 n}\right|_{1+\alpha} \leq K_{1}\left|u_{0}\right|_{1+\alpha},
$$

as $n \rightarrow \infty$. Set $v_{0 n}(x)=v_{0}(0)+\int_{0}^{x} \psi\left(y, u_{0 n}(y), w_{0 n}(y)\right) d y$ and observe that

$$
v_{0 n} \rightarrow v_{0} \text { in } C^{2}[0,1], \quad\left|v_{0 n}\right|_{2+\alpha} \leq K_{2}\left(\left|v_{0}\right|_{2+\alpha}+\left|u_{0}\right|_{1+\alpha}\right) .
$$


Let $\left(w_{n}(x, t), u_{n}(x, t)\right)$ and $\left(v_{n}(x, t), u_{n}(x, t)\right)$ be the corresponding solutions of (2.8)-(2.11) and (2.1)-(2.5), respectively; $v_{n}(x, t)$ is defined by solving (2.13) subject to $v_{n}(x, 0)=v_{0 n}(x)$. Note that, by uniqueness for (2.8)-(2.11), (2.68) and (2.13) imply that

$$
\begin{gathered}
w_{n} \rightarrow w, \partial_{x} v_{n} \rightarrow \partial_{x} v \quad \text { in } C\left(\bar{Q}_{T}\right) \\
u_{n} \rightarrow u \quad \text { in }\left[C\left(\bar{Q}_{T}\right)\right]^{N}
\end{gathered}
$$

for any fixed $T<T^{*}$.

The functions $v_{n}$ and $\omega_{n}:=\partial_{x} u_{n}$ satisfy the equations

$$
\begin{aligned}
& \partial_{t} v_{n}=A_{n}(x, t) \partial_{x}^{2} v_{n}+B_{n}(x, t) \cdot \omega_{n}+C_{n}(x, t), \\
& \partial_{t} \omega_{n}=D_{n}(x, t) \omega_{n}+E_{n}(x, t) \partial_{x}^{2} v_{n}+F_{n}(x, t),
\end{aligned}
$$

where, on account of (2.66), (2.67) and $(2.13)_{1}$, the components of $A_{n}-F_{n}$ are uniformly bounded in $C^{\alpha, \alpha / 2}\left(\bar{Q}_{T}\right)$, and, for $n$ large, $A_{n}(x, t) \geq \mu$ for some $\mu>0$. The Schauder estimates for (2.76), together with (2.74), yield

$$
\left\|v_{n}\right\|_{2+\alpha, 1+\alpha / 2} \leq K_{3}\left[\left|v_{0}\right|_{2+\alpha}+\left|u_{0}\right|_{1+\alpha}+\left\|\omega_{n}\right\|_{\alpha, \alpha / 2}+1\right] .
$$

Proceeding as in the derivation of (2.27), (2.77) implies that, on $\bar{Q}_{\tau}$ with $0<\tau \leq T$,

$$
\left\|\omega_{n}\right\|_{\alpha, \alpha / 2} \leq K_{4}\left[\left|u_{0}\right|_{1+\alpha}+\left(\tau+\tau^{1-\alpha}\right)\left\|\partial_{x}^{2} v_{n}\right\|_{\alpha, \alpha / 2}+1\right] .
$$

The constants $K_{3}$ and $K_{4}$ are independent of $n$. Combining (2.78) and (2.79), we conclude that, provided $\tau+\tau^{1-\alpha}<1 /\left(2 K_{3} K_{4}\right)$, the estimate

$$
\left\|v_{n}\right\|_{2+\alpha, 1+\alpha / 2}+\left\|\omega_{n}\right\|_{\alpha, \alpha / 2} \leq K_{5}\left(\left|v_{0}\right|_{2+\alpha}+\left|u_{0}\right|_{1+\alpha}+1\right)
$$

is valid on $\bar{Q}_{\tau}$. Also, by virtue of Lemma $2.2, w_{n}(x, t)$ satisfies $(2.41)$.

Relations (2.80) and (2.75) give $w_{x}, v_{x x} \in C^{\alpha, \alpha / 2}\left(\bar{Q}_{\tau}\right)$ and $u_{x} \in$ $\left[C^{\alpha, \alpha / 2}\left(\bar{Q}_{\tau}\right)\right]^{N}$. Also, $v_{t}=w_{x} \in C^{\alpha, \alpha / 2}\left(\bar{Q}_{\tau}\right)$ and $u_{x t}=f_{x}+f_{p} \cdot u_{x}+$ $f_{q} v_{x x} \in\left[C^{\alpha, \alpha / 2}\left(\bar{Q}_{\tau}\right)\right]^{N}$. Finally, $w(x, t)$ satisfies $(2.41)$. $\square$

3. On the competition of strain softening and strain rate dependence. The scope of this Section is to elucidate the competition 
between the destabilizing influence of strain softening and the stabilizing influence of strain rate sensitivity during the course of shearing motions and to provide quantitative criteria that determine which one prevails.

We use, as a test case, the initial-boundary value problem consisting of (1.1)-(1.3), namely,

$$
\begin{aligned}
& v_{t}=\left(\tau(u) v_{x}^{n}\right)_{x}, \\
& u_{t}=v_{x}
\end{aligned} \quad 0 \leq x \leq 1, t>0
$$

with boundary conditions $(1.9)_{S}$ and initial conditions (1.5). The initial data are taken smooth: $v_{0}(x) \in C^{2+\alpha}[0,1], u_{0}(x) \in C^{1+\alpha}[0,1]$, for some $0<\alpha<1$; compatible with the boundary conditions: $\sigma_{0}(0)=\sigma_{0}(1)=1$; and satisfying the sign restrictions

$$
u_{0}(x)>0, \quad \sigma_{0}(x)>0, \quad 0 \leq x \leq 1
$$

Henceforth, we will refer to this problem as $(\mathcal{P})_{S}$. Recall that $\tau(u)$ is a smooth function satisfying (1.4) and $n$ is a positive parameter.

The theory developed in Section 2 implies the existence of a unique solution $(v(x, t), u(x, t))$ of $(\mathcal{P})_{S}$, defined on a maximal interval of existence $[0,1] \times\left[0, T^{*}\right)$, such that $v, v_{t}, v_{x}, v_{x x}, u, u_{t}$ and $u_{x}$ are in $C^{\alpha, \alpha / 2}\left(\bar{Q}_{T}\right)$, for any $T<T^{*}$. In addition, given any compact subset $\mathcal{K}$ of $(0, \infty) \times \mathbf{R},(\sigma(x, t), u(x, t))$ escapes $\mathcal{K}$ as $t \uparrow T^{*}$, i.e., there are sequences $\left\{x_{n}\right\} \subset[0,1]$ and $\left\{t_{n}\right\}$, with $t_{n} \uparrow T^{*}$ such that $\left(\sigma\left(x_{n}, t_{n}\right), u\left(x_{n}, t_{n}\right)\right) \notin \mathcal{K}$. The identification of $(\mathcal{P})_{S}$ with $(2.1)-(2.6)$ is done by setting $w=\varphi(x, p, q)=\tau(p) q^{n}-1$. (Although $\varphi$ is not $C^{2}$ at $q=0$, it is $C^{2}$ when restricted to compact subsets of $[0,1] \times R \times(0, \infty)$; this remark, together with the results of Section 2 , provides the above statements).

Our objectives are (a) to characterize the class of functions $\tau(u)$ and parameters $n$ that guarantee global solvability of $(\mathcal{P})_{S}$, and (b) to study the behavior of solutions $(v(x, t), u(x, t))$ of $(\mathcal{P})_{S}$.

Toward this end, it is expedient to use a different formulation of (3.1). Note that, for $(x, t) \in[0,1] \times\left[0, T^{*}\right)$, a solution of $(\mathcal{P})_{S}$ satisfies

$$
\sigma(x, t)>0, \quad u_{t}(x, t)=v_{x}(x, t)>0, \quad u(x, t) \geq u_{0}(x)>0 .
$$


A simple calculation, using (1.1)-(1.3), shows that $(\sigma(x, t), u(x, t))$ is a positive solution of the reaction-diffusion system

$$
\begin{gathered}
\left(\sigma^{\frac{1}{n}}\right)_{t}=\tau(u)^{\frac{1}{n}} \sigma_{x x}+\frac{1}{n} \frac{\tau^{\prime}(u)}{\tau(u)^{1+1 / n}}\left(\sigma^{\frac{1}{n}}\right)^{2}, \\
u_{t}=\frac{\sigma^{\frac{1}{n}}}{\tau(u)^{\frac{1}{n}}},
\end{gathered}
$$

on $[0,1] \times\left[0, T^{*}\right)$. For this step and for the remainder of the Section, we assume that $(v(x, t), u(x, t))$ enjoys some additional smoothness, namely, $v_{x t}, v_{x x x}$ and $u_{x x}$ are in $C^{\alpha, \alpha / 2}\left(\bar{Q}_{T}\right)$, for any $T<T^{*}$. Such solutions are generated if we take smoother initial data, a hypothesis that can later be relaxed using density arguments (cf. Section 2). Integrating (3.5) yields

$$
\Phi(u(x, t))=\Phi\left(u_{0}(x)\right)+\int_{0}^{t} \sigma^{\frac{1}{n}}(x, \tau) d \tau,
$$

where

$$
\Phi(u)=\int_{1}^{u} \tau(\xi)^{\frac{1}{n}} d \xi
$$

An important ingredient of the forthcoming analysis lies in estimating $\sigma(x, t)$ by means of comparison principles (e.g., [13, Chapter 3, Section 7]) for the parabolic equation (3.4); in turn, $u(x, t)$ is estimated using (3.6). We state the comparison principle used as a lemma for future reference.

LEMMA 3.1. Suppose that, for any $T<T^{*}, \sigma_{1}(x, t)$ and $\sigma_{2}(x, t)$ are both in $C^{2,1}\left(\bar{Q}_{T}\right)$ with $\sigma_{1}(x, t)>0$ and $\sigma_{2}(x, t)>0, A(x, t) \in C\left(\bar{Q}_{T}\right)$ with $A(x, t)>0$, and $B(x, t) \in C\left(\bar{Q}_{T}\right)$. If, for any $T<T^{*}$,

$$
\begin{aligned}
\left(\sigma_{1}^{\frac{1}{n}}\right)_{t}-A(x, t) & \sigma_{1 x x}+B(x, t)\left(\sigma_{1}^{\frac{1}{n}}\right)^{2} \\
& \leq\left(\sigma_{2}^{\frac{1}{n}}\right)_{t}-A(x, t) \sigma_{2 x x}+B(x, t)\left(\sigma_{2}^{\frac{1}{n}}\right)^{2}, \text { on } \bar{Q}_{T} \\
\sigma_{1}(i, t) & \leq \sigma_{2}(i, t), \quad i=0,1,0 \leq t \leq T \\
\sigma_{1}(x, 0) & \leq \sigma_{2}(x, 0), \quad 0 \leq x \leq 1
\end{aligned}
$$


then

$$
\sigma_{1}(x, t) \leq \sigma_{2}(x, t), \quad 0 \leq x \leq 1,0 \leq t<T^{*}
$$

In applications of the lemma, the functions $A$ and $B$ are taken, respectively, $A=\tau(u)^{1 / n}>0$ and $B=-(1 / n)\left(\tau^{\prime}(u) / \tau(u)^{1+\frac{1}{n}}\right)>0$.

Our first theorem characterizes global solvability of $(\mathcal{P})_{S}$ in terms of the behavior of $\Phi(u)$ as $u \rightarrow \infty$.

THEOREM 3.2. Let $(v(x, t), u(x, t))$ be a classical solution of $(\mathcal{P})_{S}$ defined on a maximal interval of existence $[0,1] \times\left[0, T^{*}\right)$. Suppose that $\tau(u)$ satisfies (1.4). Then:

(i) $T^{*}=\infty$ if and only if $\Phi(\infty)=\infty$.

(ii) If $T^{*}<\infty$, then

$$
\lim _{t \rightarrow T^{*}} \sup _{0 \leq x \leq 1} u(x, t)=\infty
$$

PROOF. Let $(v(x, t), u(x, t))$ be a solution of $(\mathcal{P})_{S}$ on $[0,1] \times\left[0, T^{*}\right)$, with $T^{*}$ maximal; let $\sigma(x, t)$ be defined by (1.3). We estimate the solution in the interval of existence $[0,1] \times\left[0, T^{*}\right)$.

Under hypothesis (1.4), any positive, concave function $\Sigma(x)$ satisfies

$$
-\tau(u)^{\frac{1}{n}} \Sigma_{x x}-\frac{1}{n} \frac{\tau^{\prime}(u)}{\tau(u)^{1+\frac{1}{n}}} \Sigma^{\frac{2}{n}}>0 .
$$

If, in addition,

$$
\Sigma(x) \geq \sigma_{0}(x), \quad 0 \leq x \leq 1
$$

then applying Lemma 3.1, with comparison functions $\sigma(x, t)$ and $\Sigma(x)$, yields

$$
\sigma(x, t) \leq \Sigma(x)
$$


First, we show (ii). Assume that $T^{*}<\infty$ and, at the same time, $u(x, t)$ is bounded from above on $[0,1] \times\left[0, T^{*}\right)$, i.e.,

$$
u_{0_{-}} \leq u(x, t) \leq U_{+}<\infty ;
$$

here we used the fact that $u(x, t) \geq \inf _{0 \leq x \leq 1} u_{0}(x)=: u_{0_{-}}$. Let $s(t)$ be the solution of the initial value problem

$$
\begin{aligned}
& \frac{d}{d t} s^{\frac{1}{n}}+B_{0}\left(s^{\frac{1}{n}}\right)^{2}=0, \\
& s(0)=\sigma_{0_{-}}:=\inf _{0 \leq x \leq 1} \sigma_{0}(x),
\end{aligned}
$$

where $B_{0}=\max _{u_{0} \leq \xi \leq U_{+}}(1 / n)\left(\left|\tau^{\prime}(\xi)\right| / \tau(\xi)^{1+\frac{1}{n}}\right)$. Integrating (3.15) yields

$$
s(t)=\frac{\sigma_{0_{-}}}{\left(1+\sigma_{0_{-}}^{1 / n} B_{0} t\right)^{n}}>0 .
$$

On account of (3.15) and (3.4), the comparison functions $s(t)$ and $\sigma(x, t)$ satisfy the parabolic differential inequality $(3.8)$ on $\bar{Q}_{T}$, for any $T<T^{*}$. Using Lemma 3.1, we deduce

$$
s(t) \leq \sigma(x, t) .
$$

Estimates (3.12), (3.14) and (3.17) imply that $(\sigma(x, t), u(x, t))$ remains in some compact subset of $(0, \infty) \times \mathbf{R}$ as $t \uparrow T^{*}$. But, then the solution can be continued past $T^{*}$, which contradicts the assumption that $T^{*}$ is maximal and finite. Since $u_{t}>0$, we conclude that, if $T^{*}<\infty$ and is maximal, then (3.10) holds.

Next, we proceed to prove (i). Consider $\Phi(u)$ defined for $u \in(0, \infty)$ by (3.7). $\Phi(u)$ is increasing and invertible with an inverse function $\Phi^{-1}(\xi)$ defined for $\xi \in(\Phi(0), \Phi(\infty))$ and increasing. In case $\Phi(\infty)=$ $\infty$, combining (3.6) and (3.13), we arrive at

$$
u(x, t) \leq \Phi^{-1}\left(\Phi\left(u_{0}(x)\right)+\Sigma^{\frac{1}{n}}(x) t\right) .
$$

Then (3.14) holds for any $\bar{Q}_{T}$ with $T>0$ and, necessarily, if $T^{*}$ is maximal, then $T^{*}=\infty$. By contrast, in case $\Phi(\infty)<\infty$, (3.6) at $x=0$ or $x=1$, together with $(1.9)_{S}$, leads to

$$
\Phi(u(i, t))=\Phi\left(u_{0}(i)\right)+t, \quad i=0,1 .
$$


In turn, (3.19) implies

$$
u(i, t) \rightarrow \infty \quad \text { as } t \rightarrow T_{i},
$$

where $T_{i}=\Phi(\infty)-\Phi\left(u_{0}(i)\right)<\infty$ for $i=0,1$. Therefore, in case $\Phi(\infty)<\infty, T^{*} \leq \min \left\{T_{0}, T_{1}\right\}<\infty$.

According to Theorem 3.2, the criterion for global solvability of $(\mathcal{P})_{S}$ is the divergence of the integral $\int_{1}^{\infty} \tau(\xi)^{1 / n} d \xi=: \Phi(\infty)$. Therefore, it is the decay rate of $\tau(u)$ as $u \rightarrow \infty$ that determines global existence for $(\mathcal{P})_{S}$. The class of positive, decreasing constitutive functions $\tau(u)$ can be decomposed into two categories, depending on whether $\Phi(\infty)$ is finite or infinite. Roughly speaking, the dividing line consists of functions $\tau(u)$ that decay to zero like the power $u^{-n}$.

Next, we restrict attention to functions $\tau(u)$ such that $\Phi(\infty)=\infty$ (and, thus, $T^{*}=\infty$ ) and consider the asymptotic behavior of solutions $(v(x, t), u(x, t))$ of $(\mathcal{P})_{S}$ as $t \rightarrow \infty$. In case $\tau(u) \equiv \tau_{0}$, a constant, $\sigma(x, t)$ is a positive solution of

$$
\left(\sigma^{\frac{1}{n}}\right)_{t}=\tau_{0}^{\frac{1}{n}} \sigma_{x x}
$$

subject to the boundary conditions $(1.9)_{S}$; thus, $\sigma(x, t) \rightarrow 1$ uniformly in $x \in[0,1]$ as $t \rightarrow \infty$. The question is whether this behavior persists for positive and decreasing functions $\tau(u)$.

Two representative classes of functions $\tau(u)$ are considered: Class (H1) consists of functions that decay to a positive constant $\tau(\infty)$ at a rate dominated by a power, i.e., for some $c>0$ and $\alpha>0$,

$$
\tau(u)>\tau(\infty)>0, \quad 0<-\tau^{\prime}(u) \leq \frac{c}{u^{\alpha}}, \quad u>0 .
$$

Class (H2) consists of functions that decay to zero like a power, up to first order derivatives, i.e., for some $c>0$ and $m>0$,

$$
\frac{1}{c} \frac{1}{u^{m}} \leq \tau(u) \leq \frac{c}{u^{m}}, \quad 0<-\frac{\tau^{\prime}(u)}{\tau(u)} \leq \frac{m}{u}, \quad u>0 .
$$

Note that, to guarantee global existence for functions of class (H2), we assume $0<m \leq n$ so that $\Phi(\infty)=\infty$. Also, note that, under hypothesis (H1),

$$
\lim _{u \rightarrow \infty} \frac{\Phi(u)}{u}=\tau(\infty)^{\frac{1}{n}}
$$


while, under hypothesis (H2) for $0<m<n$,

$$
\limsup _{u \rightarrow \infty} \frac{\Phi(u)}{u^{1-\frac{m}{n}}}<\infty, \quad \liminf _{u \rightarrow \infty} \frac{\Phi(u)}{u^{1-\frac{m}{n}}}>0,
$$

and, for $m=n$,

$$
\limsup _{u \rightarrow \infty} \frac{\Phi(u)}{\ln u}<\infty, \quad \liminf _{u \rightarrow \infty} \frac{\Phi(u)}{\ln u}>0 .
$$

We prove

THEOREM 3.3. Suppose the function $\tau(u)$ belongs to either the class (H1) or the class (H2) with $0<m / n<1 / 2$. Let $(v(x, t), u(x, t))$ be a classical solution of $(\mathcal{P})_{S}$ on $[0,1] \times[0, \infty)$, corresponding to initial data $\left(v_{0}(x), u_{0}(x)\right)$ with $u_{0}(x)>0, \sigma_{0}(x)>0$ for $0 \leq x \leq 1$ and $\sigma_{0}(0)=\sigma_{0}(1)=1$. Then, for any choice of the initial data in case $0<n<2$ and, under restrictions for the data that are outlined below in case $n \geq 2$, the following hold: As $t \rightarrow \infty$,

$$
\int_{u_{0}(x)}^{u(x, t)} \tau(\xi)^{\frac{1}{n}} d \xi=t+O\left(\int_{1}^{t} s^{-\beta} d s\right)
$$

and

$$
v(x, t)=\int_{0}^{1} v_{0}(y) d y+\int_{0}^{1} \int_{y}^{x} \frac{1}{\tau(u(\xi, t))^{\frac{1}{n}}} d \xi d y+O\left(t^{-\beta+\gamma}\right)
$$

uniformly on $[0,1]$. In case (H1) holds, $\beta=\alpha>0$ and $\gamma=0$, while, in case (H2) holds, $0<\beta=(n-2 m) /(n-m)<1$ and $\gamma=m /(n-m)$.

ProOf. Let $(v(x, t), u(x, t))$ be a classical solution of $(\mathcal{P})_{S}$ defined on $\bar{Q}_{\infty}:=[0,1] \times[0, \infty)$. Then, (3.3) holds and $(\sigma(x, t), u(x, t))$ satisfies (3.4), (3.5) on $\bar{Q}_{\infty}$. We proceed to obtain an initial a priori estimate, independent of $t$, for $\sigma(x, t)$ using comparison principles. In the sequel, 
$K$ will stand for a generic constant that can be estimated in terms of the initial data and properties of the function $\tau(u)$.

Set $-\gamma(u)$ to be the quotient of the coefficient of the reaction term over the coefficient of the diffusion term in (3.4):

$$
\gamma(u)=\frac{1}{n}\left(-\frac{\tau^{\prime}(u)}{\tau(u)}\right) \frac{1}{\tau(u)^{2 / n}} .
$$

If we can find positive functions $S(x)$ and $s(x)$ defined on $[0,1]$ and satisfying, for $(x, t) \in \bar{Q}_{\infty}$, the differential inequalities

$$
\begin{gathered}
-S_{x x}+\gamma(u(x, t)) S^{2 / n} \geq 0 \\
S(x) \geq \sigma_{0}(x)
\end{gathered}
$$

and

$$
\begin{gathered}
-s_{x x}+\gamma(u(x, t)) s^{2 / n} \leq 0 \\
s(x) \leq \sigma_{0}(x),
\end{gathered}
$$

respectively, then Lemma 3.1 implies

$$
s(x) \leq \sigma(x, t) \leq S(x)
$$

for $(x, t) \in \bar{Q}_{\infty}$.

Next, we examine the possibility of finding such functions $S(x)$ and $s(x)$. Observe that, under hypothesis (H1),

$$
0<\gamma(u) \leq \frac{K_{1}}{u^{\alpha}} \leq K_{2}, \quad u \geq \inf _{0 \leq x \leq 1} u_{0}(x)
$$

while, under hypothesis (H2) for $0<m / n \leq 1 / 2$,

$$
0<\gamma(u) \leq \frac{K_{3}}{u^{1-2 \frac{m}{n}}} \leq K_{4}, \quad u \geq \inf _{0 \leq x \leq 1} u_{0}(x) .
$$

Under either of (H1) or (H2), any concave, positive function $S(x)$ satisfies (3.29) $)_{1}$. Moreover, for any choice of the initial function $\sigma_{0}(x)>0$, there is a concave function $S(x)$ such that $S(x) \geq \sigma_{0}(x)$, $0 \leq x \leq 1$. Therefore, the right-hand inequality of (3.31) is valid for all values of the parameters and choices of the initial data. 
Turn now to (3.30). If a positive function $s(x)$ satisfies

$$
-s_{x x}+\gamma_{0} s^{\frac{2}{n}} \leq 0, \quad 0 \leq x \leq 1,
$$

where $\gamma_{0}=K_{2}$ in case (H1) holds or $\gamma_{0}=K_{4}$ in case (H2) holds, $0<m / n \leq 1 / 2$, then $s(x)$ also satisfies (3.30) $)_{1}$. Consider two cases $n<2$ and $n \geq 2$.

(i) If $n<2$, the parametric family $s_{\alpha}(x)=(\alpha / 2)\left(x^{2}+1\right)$ satisfies the inequality (3.34) provided $\alpha>0$ and $\alpha^{2 / n-1} \leq 1 / \gamma_{0}$. Given any $\left(\sigma_{0}(x), u_{0}(x)\right)$ both positive on $[0,1]$, we fix $\gamma_{0}$ (which depends on $u_{0}(x)$ ) and choose $\alpha$ sufficiently small so that $s_{\alpha}(x) \leq \sigma_{0}(x)$ and (3.34) is fulfilled for $x \in[0,1]$. Then, $s_{\alpha}(x)$ satisfies (3.30), and the left-hand side of (3.31) is established.

(ii) If $n \geq 2$, a function $s(x)$ satisfying (3.34) and $(3.30)_{2}$ can only be found for restricted choices of the data $\left(\sigma_{0}(x), u_{0}(x)\right)$. For instance, given $\sigma_{0}(x)$, find the largest $\alpha>0$ such that $s_{\alpha}(x)=(\alpha / 2)\left(x^{2}+1\right) \leq$ $\sigma_{0}(x)$. With this $\alpha$ fixed, $s_{\alpha}(x)$ satisfies (3.34) provided $\gamma_{0} \leq \alpha^{1-2 / n}$. In view of the choice of $\gamma_{0}$ and (3.32), (3.33), this imposes a restriction on $u_{0}(x)$ and/or the function $\tau(u)$. If these restrictions are satisfied, then the left-hand inequality of (3.31) holds.

Henceforth, we restrict attention to the cases when (3.31) holds. Using (3.31), (3.6) yields

$$
\frac{1}{K_{5}} t \leq \Phi(u(x, t))-\Phi\left(u_{0}(x)\right) \leq K_{5} t, \quad t>0 .
$$

Combining (3.3) and (3.35) with (3.22) or (3.23), we conclude that, under hypothesis (H1),

$$
\frac{1}{K_{6}}(t+1) \leq u(x, t) \leq K_{6}(t+1),
$$

while, under hypothesis (H2) with $0<m / n \leq 1 / 2$,

$$
\frac{1}{K_{7}}(t+1)^{\frac{n}{n-m}} \leq u(x, t) \leq K_{7}(t+1)^{\frac{n}{n-m}} .
$$

In view of (1.1) and $(1.9)_{S}$, we have the identity

$$
\frac{1}{2} \frac{d}{d t} \int_{0}^{1} v_{t}^{2} d x+\int_{0}^{1} \sigma_{t} v_{x t} d x=0 .
$$


Using (3.38), together with (3.4) and (1.1),

$$
\frac{1}{2} \frac{d}{d t} \int_{0}^{1} v_{t}^{2} d x+n \int_{0}^{1} \tau(u)^{\frac{1}{n}} \sigma^{1-\frac{1}{n}} v_{x t}^{2} d x=\int_{0}^{1}\left(-\frac{\tau^{\prime}(u)}{\tau(u)}\right) \frac{\sigma^{1+\frac{1}{n}}}{\tau(u)^{\frac{1}{n}}} v_{x t} d x,
$$

which, together with Schwarz's inequality, implies

$$
\frac{1}{2} \frac{d}{d t} \int_{0}^{1} v_{t}^{2} d x+\frac{n}{2} \int_{0}^{1} \tau(u)^{\frac{1}{n}} \sigma^{1-\frac{1}{n}} v_{x t}^{2} d x \leq \frac{1}{2 n} \int_{0}^{1}\left(\frac{\tau^{\prime}(u)}{\tau(u)}\right)^{2} \frac{\sigma^{1+\frac{3}{n}}}{\tau(u)^{\frac{3}{n}}} d x
$$

Also, the calculus identity

$$
\sigma_{x}(x, t)=\int_{0}^{1} \sigma_{x}(y, t) d y+\int_{0}^{1} \int_{y}^{x} \sigma_{x x}(\xi, t) d \xi
$$

together with (1.1), (1.9) $S$ and Schwarz's inequality, leads to

$$
v_{t}^{2}(x, t) \leq \int_{0}^{1} v_{x t}^{2}(\xi, t) d \xi
$$

Suppose first that $(\mathrm{H} 2)$ holds and $0<m / n \leq 1 / 2$. Then, combining (3.31), (3.37) and the inequalities in (H2) with (3.40) and (3.42), we arrive at the differential inequality

$$
\frac{d}{d t} \int_{0}^{1} v_{t}^{2} d x+\frac{1}{K_{8}}(t+1)^{-\frac{m}{n-m}} \int_{0}^{1} v_{t}^{2} d x \leq K_{9}(t+1)^{\frac{3 m-2 n}{n-m}}
$$

If $0<m / n<1 / 2$, then integrating (3.43) yields

$$
\int_{0}^{1} v_{t}^{2}(x, t) d x \leq K_{10}(t+1)^{\frac{4 m-2 n}{n-m}}
$$

however, if $m / n=1 / 2$, then (3.43) does not provide any decay. Relation (3.44), in conjunction with the Poincaré inequality

$$
(\sigma(x, t)-1)^{2} \leq \int_{0}^{1} \sigma_{x}^{2}(\xi, t) d \xi
$$

and (1.1), yields (3.25) when (H2) holds. 
When (H1) holds, the same sequence of steps using (3.36) and (H1) in the place of (3.37) and (H2) lead to the differential inequality

$$
\frac{d}{d t} \int_{0}^{1} v_{t}^{2} d x+\frac{1}{K_{11}} \int_{0}^{1} v_{t}^{2} d x \leq K_{12}(t+1)^{-2 \alpha},
$$

which, once integrated, yields

$$
\int_{0}^{1} v_{t}^{2}(x, t) d x \leq K_{13}(t+1)^{-2 \alpha} .
$$

Combining (3.45) and (3.47), we arrive at (3.25) in case (H1) holds.

To show (3.26), observe that (3.6), (3.7), Poincaré's inequality, (3.31) and (1.1) yield

$$
\begin{aligned}
\left|\int_{u_{0}(x)}^{u(x, t)} \tau(\xi)^{\frac{1}{n}} d \xi-t\right| & \leq \int_{0}^{t}\left|\sigma^{\frac{1}{n}}(x, \tau)-1\right| d \tau \\
& \leq K_{14} \int_{0}^{t}\left(\int_{0}^{1} v_{t}^{2}(x, \tau) d x\right)^{1 / 2} d \tau .
\end{aligned}
$$

Using (3.48), together with (3.44) or (3.47) in cases (H2) or (H1), respectively, we deduce (3.26).

Finally, the identities

$$
v(x, t)=\int_{0}^{1} v(y, t) d y+\int_{0}^{1} \int_{y}^{x} \frac{\sigma^{\frac{1}{n}}(\xi, t)}{\tau(u(\xi, t))^{\frac{1}{n}}} d \xi d y
$$

and

$$
\int_{0}^{1} v(y, t) d y=\int_{0}^{1} v_{0}(y) d y
$$

(by (1.1) and $\left.(1.9)_{S}\right)$, together with Poincaré's inequality and (3.31), imply

$$
\begin{aligned}
\mid v(x, t)-\int_{0}^{1} v_{0}(y) d y & -\int_{0}^{1} \int_{y}^{x} \frac{1}{\tau(u(\xi, t))^{\frac{1}{n}}} d \xi d y \mid \\
& \leq \int_{0}^{1} \frac{\left|\sigma^{\frac{1}{n}}-1\right|}{\tau(u)^{\frac{1}{n}}} d x \\
& \leq K_{15}\left(\int_{0}^{1} v_{t}^{2} d x\right)^{1 / 2}\left(\int_{0}^{1} \frac{1}{\tau(u)^{\frac{1}{n}}} d x\right) .
\end{aligned}
$$


Using (3.47) in case (H1) holds, or (3.44) and (3.37) in case (H2) holds, we arrive at $(3.27)$.

Between the class of functions $\tau(u)$ satisfying $\Phi(\infty)<\infty$, for which solutions of $(\mathcal{P})_{S}$ blow up in finite time, and the class of $\tau(u)$ satisfying (H2) with $0<m / n<1 / 2$, for which solutions of $(\mathcal{P})_{S}$ behave asymptotically like in (3.21) (cf. (3.25)), there remains a gap to be analyzed. For a power law

$$
\tau(u)=u^{-m}
$$

the gap corresponds to the powers $1 / 2 \leq m / n \leq 1$.

In the sequel we consider the power law (3.52) with $m / n \geq 1 / 2$ and analyze the behavior of solutions of $(\mathcal{P})_{S}$. Let $(v(x, t), u(x, t))$ be such a solution defined on $[0,1] \times\left[0, T^{*}\right)$; here $T^{*}=+\infty$ if $1 / 2 \leq m / n \leq 1$ and $T^{*}<+\infty$ if $m / n>1$. The function $\sigma(x, t)$ satisfies (3.4) with $\tau(u)$ as in (3.52), while (3.6) yields

$$
u(x, t)^{1-\frac{m}{n}}=u_{0}(x)^{1-\frac{m}{n}}+\left(1-\frac{m}{n}\right) \int_{0}^{t} \sigma^{\frac{1}{n}}(x, \tau) d \tau,
$$

in case $m / n \neq 1$, and

$$
\ln u(x, t)=\ln u_{0}(x)+\int_{0}^{t} \sigma^{\frac{1}{n}}(x, \tau) d \tau
$$

in case $m / n=1$.

We examine the class of solutions of the differential inequality (3.29). Note that, by virtue of (3.28), (3.52) and (3.3), for $m / n \geq 1 / 2$,

$$
\gamma(u(x, t))=\frac{m}{n} u(x, t)^{2 \frac{m}{n}-1} \geq \frac{m}{n} u_{0}(x)^{2 \frac{m}{n}-1} .
$$

Then Lemma 3.1 implies

LEMMA 3.4. Let $m / n \geq 1 / 2$. If $S(x)$ is a smooth, positive function satisfying, for $x \in[0,1]$,

$$
\begin{gathered}
-S_{x x}(x)+\gamma_{0}(x) S^{\frac{2}{n}}(x) \geq 0 \\
S(x) \geq \sigma_{0}(x),
\end{gathered}
$$


where $\gamma_{0}(x):=(m / n) u_{0}(x)^{2 \frac{m}{n}-1}$, then

$$
\sigma(x, t) \leq S(x), \quad 0 \leq x \leq 1,0 \leq t<T^{*} .
$$

If $m / n>1 / 2$, given any $S(x) \geq \sigma_{0}(x)>0$, (3.56) can always be satisfied by choosing $u_{0}(x)>0$ appropriately. Namely,

$$
\frac{m}{n} u_{0}(x)^{2 \frac{m}{n}-1} \geq \frac{S_{x x}(x)}{S^{\frac{2}{n}}(x)} .
$$

In particular, $\sigma_{0}(x)$ can be used as a choice for $S(x)$ for restricted choices of $u_{0}(x)$.

If $m / n=1 / 2$, then $S(x) \geq \sigma_{0}(x)>0$ will satisfy (3.56) provided

$$
\frac{1}{2} \geq \frac{S_{x x}(x)}{S^{\frac{2}{n}}(x)}
$$

In light of the above remark, we study the family of potential choices

$$
S_{\alpha}(x)=1+\alpha x(x-1), \quad 0 \leq x \leq 1,
$$

where $0<\alpha<4$. Note that $S_{\alpha}(x)$ is convex and attains its minimum $S_{\alpha}(1 / 2)=1-\alpha / 4>0$. Also, $S_{\alpha}(x) \rightarrow 1$ uniformly on $[0,1]$ as $\alpha \rightarrow 0$.

(a) Case $m / n>1 / 2$. If the initial data $\left(u_{0}(x), \sigma_{0}(x)\right)$ are restricted so as to satisfy

$$
\sigma_{0}(x) \leq S_{\alpha}(x), \quad u_{0}(x) \geq u_{\alpha}:=\left[\frac{n}{m} \frac{2 \alpha}{\left(1-\frac{\alpha}{4}\right)^{\frac{2}{n}}}\right]^{\frac{n}{2 m-n}}, \quad 0 \leq x \leq 1,
$$

then Lemma 3.4, in conjunction with (3.58), implies

$$
\sigma(x, t) \leq S_{\alpha}(x), \quad 0 \leq x \leq 1,0 \leq t<T^{*}
$$

Observe that $u_{\alpha} \rightarrow 0$ as $\alpha \rightarrow 0$.

Consider now the problem $(\mathcal{P})_{S}$ with initial data $\left(\sigma_{0}(x), u_{0}(x)\right)=$ $\left(S_{\alpha}(x), u_{\alpha}\right)$, for some $0<\alpha<4$. Let $\left(\sigma_{\alpha}(x, t), u_{\alpha}(x, t)\right)$ be the corresponding solution; it is defined on $[0,1] \times\left[0, T^{*}\right)$. Also,

$$
\sigma_{\alpha}(x, t) \leq S_{\alpha}(x)=1+\alpha x(x-1)
$$

for $(x, t) \in[0,1] \times\left[0, T^{*}\right)$. 
We consider three separate regions:

(i) $(1 / 2<m / n<1)$ Here $T^{*}=+\infty$. In addition, (3.63) and (3.53) yield

$$
u_{\alpha}(x, t) \leq\left[u_{\alpha}^{1-\frac{m}{n}}+\left(1-\frac{m}{n}\right) S_{\alpha}^{\frac{1}{n}}(x) t\right]^{\frac{n}{n-m}}
$$

for $0 \leq x \leq 1,0 \leq t<\infty$. Note that, since $S_{\alpha}(0)=S_{\alpha}(1)=1$, the boundary condition $(1.9)_{S}$, together with (3.53), implies that (3.64) is, in fact, an equality at $x=0$ and $x=1$. A comparison of (3.25) and (3.26) with (3.63) and (3.64) reveals the drastic difference in the behavior of solutions across the parameter values $m / n=1 / 2$. In particular, $\sigma_{\alpha}(x, t)$ does not converge to 1 as $t \rightarrow \infty$, and spatial nonuniformities of the strain $u(x, t)$ develop and persist in time. This is the case no matter how close to the constant function 1 the initial state $S_{\alpha}(x)$ is. The diffusion is, in this case, too weak to uniformize the solution. by

(ii) $(m / n=1)$ The situation is similar to part (i) with (3.64) replaced

$$
u_{\alpha}(x, t) \leq u_{\alpha} \exp \left\{S_{\alpha}^{\frac{1}{n}}(x) t\right\},
$$

by virtue of (3.54).

(iii) $(m / n>1)$ Here $T^{*}<+\infty$. The solution $\left(\sigma_{\alpha}(x, t), u_{\alpha}(x, t)\right)$ satisfies, on $[0,1] \times\left[0, T^{*}\right)$, the bounds $(3.63)$ and

$$
u_{\alpha}(x, t) \leq\left[u_{\alpha}^{1-\frac{m}{n}}-\left|1-\frac{m}{n}\right| S_{\alpha}^{\frac{1}{n}}(x) t\right]^{-\frac{n}{m-n}} .
$$

The bound on the right-hand side of (3.66) blows up for the first time at the boundary points $x=0$ and $x=1$ as $t \rightarrow T_{\mathrm{cr}}:=\frac{1}{\left|1-\frac{m}{n}\right|} u_{\alpha}^{1-\frac{m}{n}}$. Then (3.10) and (3.66) imply that $T^{*} \geq T_{\mathrm{cr}}$. However, since $S_{\alpha}(0)=$ $S_{\alpha}(1)=1,(3.66)$ is, in fact, an equality at $x=0$ and $x=1$ and, thus, $T^{*}=T_{\mathrm{cr}}$. The function $u_{\alpha}(x, t)$ blows up exactly at the boundary points $x=0$ and $x=1$ as $t \rightarrow T_{\mathrm{cr}}=T^{*}$; in blowing up it satisfies (3.66) and appears like two shear bands located at the boundaries $x=0$ and $x=1$. Moreover, as $t \rightarrow T^{*}$, the function $\sigma_{\alpha}(x, t)$ obeys the bound (3.63), while

$$
\partial_{t} u_{\alpha}(x, t)=\partial_{x} v_{\alpha}(x, t) \leq S_{\alpha}^{\frac{1}{n}}(x)\left[u_{\alpha}^{1-\frac{m}{n}}-\left|1-\frac{m}{n}\right| S_{\alpha}^{\frac{1}{n}}(x) t\right]^{-\frac{m}{m-n}}
$$

with an equality at $x=0$ and $x=1$. 
Note that, in the above cases, any $0<\alpha<4$ can be chosen. Also, by choosing other types of functions $S(x)$ in the place of (3.60), the nonuniformities that develop near the boundary can be made very strong, at the expense of restrictions on the initial data (cf. (3.58)).

(b) Case $m / n=1 / 2$. If $\alpha$ is small enough so that $2 \alpha \leq$ $(1 / 2)(1-\alpha / 4)^{2 / n}$, then $S_{\alpha}(x)$ satisfies (3.59). Consider initial data $\left(S_{\alpha}(x), u_{0}(x)\right)$, with $\alpha$ small and $u_{0}(x)>0$ but otherwise unrestricted. Let $\left(\sigma_{\alpha}(x, t), u_{\alpha}(x, t)\right)$ be the corresponding solution of $(\mathcal{P})_{S}$; it is defined on $[0,1] \times[0, \infty)$. On account of $(3.60)$ and Lemma 3.4, $\sigma_{\alpha}(x, t)$ satisfies (3.63) and the uniform state $\sigma \equiv 1$ again loses stability, as is the situation in the case $m / n>1 / 2$.

4. The power law and scale invariance. For the particular choice $\tau(u)=1 / u^{m}$, the constitutive relation (1.3) takes the form of the power law

$$
\sigma=\frac{1}{u^{m}} v_{x}^{n},
$$

where $m, n$ are positive parameters. Under (4.1), (1.1)-(1.2) read

$$
\begin{aligned}
& v_{t}=\left(\frac{1}{u^{m}} v_{x}^{n}\right)_{x} \\
& u_{t}=v_{x}
\end{aligned}
$$

and, correspondingly, (3.4)-(3.5) take the form

$$
\begin{aligned}
\left(\sigma^{\frac{1}{n}}\right)_{t} & =u^{-\frac{m}{n}} \sigma_{x x}-\frac{m}{n} u^{\frac{m}{n}-1} \sigma^{\frac{2}{n}} \\
u_{t} & =u^{\frac{m}{n}} \sigma^{\frac{1}{n}} .
\end{aligned}
$$

The power structure of the system (4.2) induces the following scaling property: If $(v(x, t), u(x, t))$ is a solution of $(4.2)$ on $\mathbf{R} \times(0, \infty)$, then $\left(v_{\lambda}(x, t), u_{\lambda}(x, t)\right)$, defined by

$$
\begin{gathered}
v_{\lambda}(x, t)=\lambda^{\frac{\delta}{\alpha}} v\left(\lambda x, \lambda^{-\frac{1}{\alpha}} t\right) \\
u_{\lambda}(x, t)=\lambda^{\frac{\alpha+\delta+1}{\alpha}} u\left(\lambda x, \lambda^{-\frac{1}{\alpha}} t\right),
\end{gathered}
$$


where $\lambda>0$ and $\delta, \alpha$ are any constants with

$$
-m(\alpha+\delta+1)+n(\alpha+\delta)+\alpha-\delta+1=0,
$$

is also a solution of $(4.2)$ on $\mathbf{R} \times(0, \infty)[\mathbf{1 6}]$. As a consequence,

$$
\sigma_{\lambda}(x, t)=\lambda^{\frac{\delta-\alpha-1}{\alpha}} \sigma\left(\lambda x, \lambda^{-\frac{1}{\alpha}} t\right)
$$

and $\left(\sigma_{\lambda}(x, t), u_{\lambda}(x, t)\right)$ satisfies $(4.3)$ on $\mathbf{R} \times(0, \infty)$ for any $\lambda>0$. Therefore, the systems (4.2) and (4.3) are invariant under the group of stretching transformations $T_{\alpha, \delta}$ :

$$
\begin{gathered}
x \rightarrow \lambda x, \quad t \rightarrow \lambda^{-\frac{1}{\alpha}} t, \quad v \rightarrow \lambda^{\frac{\delta}{\alpha}} v, \quad u \rightarrow \lambda^{\frac{\alpha+\delta+1}{\alpha}} u, \\
\sigma \rightarrow \lambda^{\frac{\delta-\alpha-1}{\alpha}} \sigma, \quad 0<\lambda<\infty
\end{gathered}
$$

with $\alpha, \delta$ constrained by (4.6).

The system (4.2) admits a special class of solutions describing uniform shearing

$$
\bar{v}(x, t)=x, \quad \bar{u}(x, t)=t+u_{0}
$$

They correspond to initial data $\bar{v}_{0}(x)=x$ and $\bar{u}_{0}(x)=u_{0}$, where $u_{0}$ is an arbitrary positive constant. For the special choice $u_{0}=0$, $(x, t)$ is a self-similar solution under the transformation $T_{\alpha, \delta}$ with $\alpha=-\delta=(m-1) / 2$.

Consider now the initial-boundary value problem consisting of (4.2) on $[0,1] \times\{t>0\}$ with boundary conditions $(1.9)_{V}$ and initial conditions (1.5). Suppose that the initial data are smooth, $v_{0}(x) \in$ $C^{2+\alpha}[0,1], u_{0}(x) \in C^{1+\alpha}[0,1]$, for some $0<\alpha<1$, are compatible with the boundary data, and satisfy the sign restrictions $\sigma_{0}(x)>0$, $u_{0}(x)>0,0 \leq x \leq 1$. We will refer to this problem as $(\mathcal{P})_{V}$ (including the assumptions on the initial data).

The existence theory developed in Section 2 implies that $(\mathcal{P})_{V}$ admits a unique classical solution defined on a maximal interval of existence $[0,1] \times\left[0, T^{*}\right)$. Moreover, if $T^{*}<+\infty$, given any compact subset $\mathcal{K}$ of $(0, \infty) \times(0, \infty),(\sigma(x, t), u(x, t))$ escapes $\mathcal{K}$ as $t \uparrow T^{*}$. Also, for $(x, t) \in[0,1] \times\left[0, T^{*}\right)$,

$$
\sigma(x, t)>0, \quad u_{t}(x, t)>0, \quad u(x, t) \geq u_{0}(x) .
$$


The uniform shearing solution (4.9) is a special solution of $(\mathcal{P})_{V}$ for initial data $\left(x, u_{0}\right)$. Our objective is to study the stability of this solution. To this end, we use a transformation, motivated by the scaling properties of (4.2), to obtain a system that admits invariant regions [4]. A similar idea has been independently pursued by Bertsch, Peletier and Verduyn Lunel $[\mathbf{1}]$ for a related system.

We prove

TheOREM 4.1. Suppose $m<n$. There exists a unique classical solution $(v(x, t), u(x, t))$ of $(\mathcal{P})_{V}$ defined on $[0,1] \times[0, \infty)$ such that $v, v_{x}, v_{t}, v_{x x}, u, u_{x}$ and $u_{t}$ are in $C^{\alpha, \alpha / 2}\left(\bar{Q}_{T}\right)$, for any $T>0$. Moreover, if $m<\min \{n, 1\}$, as $t \rightarrow \infty$,

$$
\begin{gathered}
v_{x}(x, t)=1+O\left(t^{\beta-1}\right) \\
u(x, t)=t+O\left(t^{\beta}\right)
\end{gathered}
$$

and

$$
\sigma(x, t)=t^{-m}\left(1+O\left(t^{\beta-1}\right)\right),
$$

uniformly on $[0,1]$, with $\beta=\max \{m / n, m\}<1$.

PROOF. Introduce the transformations

$$
\begin{gathered}
v(x, t)=V(x, s(t)) \\
u(x, t)=(t+1) U(x, s(t)) \\
\sigma(x, t)=(t+1)^{-m} \Sigma(x, s(t))
\end{gathered}
$$

with

$$
s(t)=\ln (t+1),
$$

which are motivated by the form of the uniform shearing solutions and the scaling invariance (4.8). Relations (4.14)-(4.16) and (4.1) induce

$$
\Sigma=\frac{1}{U^{m}} V_{x}^{n} .
$$


Moreover, since $(\sigma(x, t), u(x, t))$ satisfies $(4.3)$ and

$$
\sigma_{x}(0, t)=\sigma_{x}(1, t)=0, \quad t>0,
$$

it follows that $(\Sigma(x, s), U(x, s))$ solve the system of reaction-diffusion equations

$$
\begin{aligned}
& \Sigma_{s}=n e^{(1-m) s} U^{-\frac{m}{n}} \Sigma^{1-\frac{1}{n}} \Sigma_{x x}-m \frac{\Sigma}{U^{1-\frac{m}{n}}}\left(\Sigma^{\frac{1}{n}}-U^{1-\frac{m}{n}}\right) \\
& U_{s}=U^{\frac{m}{n}}\left(\Sigma^{\frac{1}{n}}-U^{1-\frac{m}{n}}\right)
\end{aligned}
$$

subject to boundary conditions

$$
\Sigma_{x}(0, s)=\Sigma_{x}(1, s)=0, \quad s>0,
$$

and initial conditions

$$
\Sigma(x, 0)=\sigma_{0}(x), \quad U(x, 0)=u_{0}(x), \quad 0 \leq x \leq 1 .
$$

If $0<m / n<1$, the theory of Chueh, Conley and Smoller [4] guarantees that (4.20) admits positively invariant rectangles of arbitrary size in the first quadrant $\left\{(\Sigma, U) \in \mathbf{R}^{2}: \Sigma>0, U>0\right\}$. They are centered around the line $\Sigma=U^{n-m}$ and look like that in Figure 1. Given initial data $\sigma_{0}(x)>0, u_{0}(x)>0$, let $U_{-}, U_{+}, \Sigma_{-}$and $\Sigma_{+}$be the defining coordinates of the smallest invariant rectangle containing $\left(\sigma_{0}(x), u_{0}(x)\right)$, $0 \leq x \leq 1$. Then

$$
\Sigma_{-} \leq \Sigma(x, s) \leq \Sigma_{+}, \quad U_{-} \leq U(x, s) \leq U_{+} .
$$

In turn, (4.15), (4.16), (4.14) and (4.18), in conjunction with (4.23), yield

$$
\begin{aligned}
U_{-}(t+1) & \leq u(x, t) \leq U_{+}(t+1), \\
\Sigma_{-}(t+1)^{-m} & \leq \sigma(x, t) \leq \Sigma_{+}(t+1)^{-m}, \\
\Sigma_{-}^{\frac{1}{n}} U_{-}^{\frac{m}{n}} & \leq v_{x}(x, t) \leq \Sigma_{+}^{\frac{1}{n}} U_{+}^{\frac{m}{n}} .
\end{aligned}
$$

The first implication of (4.24)-(4.26) is that, for $0<m / n<1$, the functions $(\sigma(x, t), u(x, t))$ remain in a compact subset of $(0, \infty) \times(0, \infty)$ for any finite time. Thus, $T^{*}=+\infty$, that is, solutions $(v(x, t), u(x, t))$ 


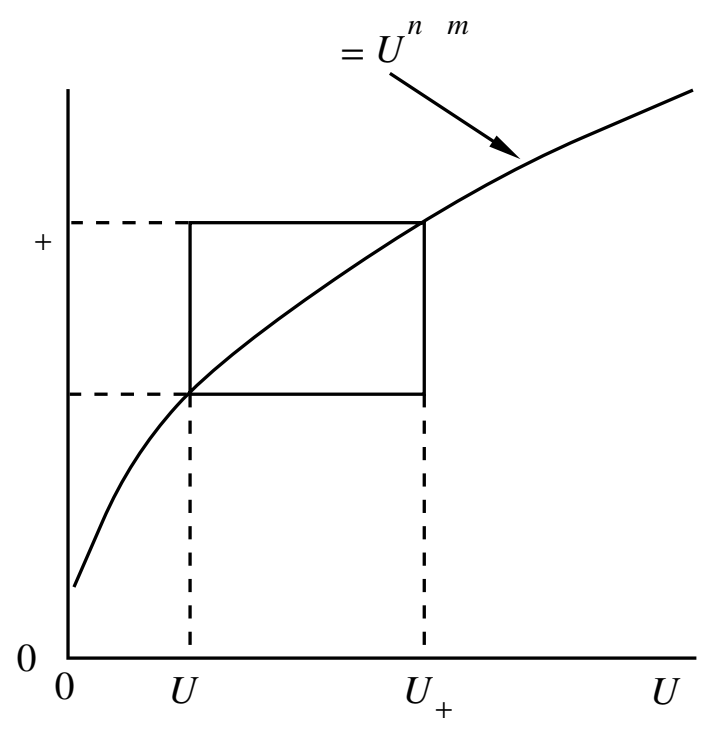

FIGURE 1. Invariant regions for (4.20).

of $(\mathcal{P})_{V}$ are globally defined. In addition, (4.24)-(4.26) provide preliminary information on the time evolution of solutions. They are supplemented below with parabolic-type energy estimates to establish the stated asymptotic behavior. In what follows, $K$ will stand for a generic constant that depends only on the data and the parameters $m$ and $n$.

Our first goal is to estimate the $L^{2}$-norm of $v_{t}$. Toward this end, differentiate (1.1) with respect to $t$ and use (4.1) and (1.2) to obtain

$$
v_{t t}=\left(\frac{1}{u^{m}} n v_{x}^{n-1} v_{x t}-\frac{m}{u^{m+1}} v_{x}^{n+1}\right)_{x} .
$$

We multiply (4.27) by $v_{t}$ and integrate by parts over [0,1], using $(1.9)_{V}$, to arrive at

$$
\frac{1}{2} \frac{d}{d t} \int_{0}^{1} v_{t}^{2} d x+n \int_{0}^{1} \frac{v_{x}^{n-1}}{u^{m}} v_{x t}^{2} d x=m \int_{0}^{1} \frac{v_{x}^{n+1}}{u^{m+1}} v_{x t} d x .
$$

On account of (4.24), (4.26) and Schwarz's inequality, (4.28) yields

$$
\frac{d}{d t} \int_{0}^{1} v_{t}^{2} d x+\frac{1}{K_{1}}(t+1)^{-m} \int_{0}^{1} v_{x t}^{2} d x \leq K_{1}(t+1)^{-m-2} .
$$


Finally, combining (4.29) with the Poincaré inequality

$$
v_{t}^{2}(x, t) \leq \int_{0}^{1} v_{x t}^{2}(x, t) d x,
$$

we arrive at the differential inequality

$$
\frac{d}{d t} \int_{0}^{1} v_{t}^{2} d x+\frac{1}{K_{1}}(t+1)^{-m} \int_{0}^{1} v_{t}^{2} d x \leq K_{1}(t+1)^{-m-2} .
$$

Integrating (4.31), we deduce

$$
\begin{aligned}
\int_{0}^{1} v_{t}^{2}(x, t) d x \leq & \left(\int_{0}^{1} v_{t}^{2}(x, 0) d x\right) \exp \left\{-\frac{1}{K_{1}} \int_{0}^{t}(s+1)^{-m} d s\right\} \\
& +K_{1} \int_{0}^{t}(s+1)^{-m-2} \exp \left\{-\frac{1}{K_{1}} \int_{s}^{t}(\tau+1)^{-m} d \tau\right\} d s .
\end{aligned}
$$

In case $m<1$, L'Hopital's rule implies

$$
\lim _{t \rightarrow \infty} \frac{\int_{0}^{t}(s+1)^{-m-2} \exp \left\{\frac{1}{K_{1}} \int_{0}^{s}(\tau+1)^{-m} d \tau\right\} d s}{(t+1)^{-2} \exp \left\{\frac{1}{K_{1}} \int_{0}^{t}(\tau+1)^{-m} d \tau\right\}}=K_{1} .
$$

In view of (4.33), (4.31) yields, for $0<m<1$,

$$
\int_{0}^{1} v_{t}^{2}(x, t) d x \leq K_{2}(t+1)^{-2}
$$

By contrast, if $m>1,(4.32)$ does not provide decay for the $L^{2}$-norm of $v_{t}$. Finally, if $m=1$, the decay rate depends on the coefficient $K_{1}$ in (4.31).

Equations (4.3) $)_{2}$ and (1.1) readily imply

$$
u^{-\frac{m}{n}}(x, t) u_{x}(x, t)=u_{0}^{-\frac{m}{n}}(x) u_{0 x}(x)+\frac{1}{n} \int_{0}^{t} \sigma^{\frac{1}{n}-1}(x, \tau) v_{t}(x, \tau) d \tau .
$$

Two cases are considered: (i) If $n \neq 1$, then, by virtue of (4.24), (4.25) and $(4.34),(4.35)$ yields

$$
\begin{aligned}
\int_{0}^{1}\left|u_{x}(x, t)\right| d x \leq & K_{3}(t+1)^{\frac{m}{n}} \\
& +K_{4}(t+1)^{\frac{m}{n}} \int_{0}^{t}(\tau+1)^{-\frac{m}{n}+m}\left(\int_{0}^{1} v_{t}^{2}(x, \tau) d x\right)^{1 / 2} d \tau \\
\leq & K_{5}(t+1)^{\frac{m}{n}}+K_{6}(t+1)^{m} .
\end{aligned}
$$


(ii) If $n=1$, then (4.35) reads

$$
u^{-m}(x, t) u_{x}(x, t)=u_{0}^{-m}(x) u_{0 x}(x)+v(x, t)-v_{0}(x) .
$$

Using (4.24), together with the maximum principle for $(4.2)_{1}$, we again arrive at (4.36). Combining (4.36) with the identities

$$
\begin{aligned}
u(x, t)-\int_{0}^{1} u(y, t) d y & =\int_{0}^{1} \int_{y}^{x} u_{x}(\xi, t) d \xi d y \\
\int_{0}^{1} u(y, t) d y & =t+\int_{0}^{1} u_{0}(y) d y
\end{aligned}
$$

obtains (4.12).

Next, use the identity,

$$
n v_{x}^{n-1} v_{x x}=u^{m} v_{t}+\frac{m}{u} u_{x} v_{x}^{n}
$$

in conjunction with $(4.26),(4.24),(4.34)$, and (4.36) to deduce

$$
\begin{aligned}
\int_{0}^{1}\left|v_{x x}(x, t)\right| d x \leq & K_{7}(t+1)^{m}\left(\int_{0}^{1} v_{t}^{2}(x, t) d x\right)^{1 / 2} \\
& +\frac{K_{8}}{t+1} \int_{0}^{1}\left|u_{x}(x, t)\right| d x \\
\leq & K_{9}(t+1)^{m-1}+K_{10}(t+1)^{\frac{m}{n}-1} .
\end{aligned}
$$

Then (4.11) follows by virtue of (4.40) and the Poincaré inequality

$$
\left|v_{x}(x, t)-1\right| \leq \int_{0}^{1}\left|v_{x x}(x, t)\right| d x
$$

Finally, to show (4.13), note that, on account of (4.11), (4.12), as $t \rightarrow \infty$,

$$
\begin{gathered}
v_{x}^{n}(x, t)=1+O\left(t^{\beta-1}\right) \\
u^{-m}(x, t)=t^{-m}\left(1+O\left(t^{\beta-1}\right)\right),
\end{gathered}
$$

where $\beta=\max \{m / n, m\}<1$. Combining (4.1) with (4.42) and (4.43) gives (4.13). 
To shed some light on the relevance of the constraint $m<1$, consider the case $1<m<n$, and observe that (4.24) implies

$$
U_{+}^{-m}(t+1)^{-m} \leq u^{-m}(x, t) \leq U_{-}^{-m}(t+1)^{-m} .
$$

Thus, the diffusion coefficient in $(4.2)_{1}$ decays like $(t+1)^{-m}$.

Consider the problem

$$
v_{t}=a(t)\left(v_{x}^{n}\right)_{x}
$$

on $[0,1] \times[0, \infty)$, subject to $(1.9)_{V}$ and $v(x, 0)=v_{0}(x)$ with $v_{0 x}(x)>0$. The change of variables

$$
\begin{gathered}
V(x, s(t))=v(x, t) \\
s(t)=\int_{0}^{t} a(\tau) d \tau
\end{gathered}
$$

suggests that $V(x, s)$ satisfies

$$
V_{s}=\left(V_{x}^{n}\right)_{x}
$$

subject to the same initial and boundary conditions. For $a(t)=$ $(t+1)^{-m}$, we have that $s_{\infty}:=\lim _{t \rightarrow \infty} s(t)$ is infinite for $m \leq 1$, but finite for $m>1$. Also,

$$
\lim _{t \rightarrow \infty} v(x, t)=\lim _{s \rightarrow s_{\infty}} V(x, s) .
$$

If $s_{\infty}=+\infty$, then $\lim _{t \rightarrow \infty} v(x, t)=x$; however, if $s_{\infty}<+\infty$, in general, this will no longer be true.

\section{REFERENCES}

1. M. Bertsch, L.A. Peletier and S.M. Verduyn Lunel, The effect of temperature dependent viscocity on shear flow of incompressible fluids, SIAM J. Math. Anal., to appear.

2. N. Charalambakis, Stabilité asymptotique lorsque $t \rightarrow \infty$ en thermoviscoelasticité et thermoplasticité, in Nonlinear partial differential equations and their applications: Collège de France Seminar (H. Brezis and J.-L. Lions, eds.), Vol. IX, Pitman Res. Notes in Math., No. 181, Longman Sci. Tech., Essex, England, 1988. 
3. N. Charalambakis and F. Murat, Weak solutions to the initial-boundary value problem for the shearing of non-homogeneous thermoviscoplastic materials, Proc. Roy. Soc. Edinburgh Sect. A 113 (1989), 257-265.

4. K. Chueh, C. Conley and J. Smoller, Positively invariant regions for systems of nonlinear diffusion equations, Indiana Univ. Math. J. 26 (1977), 372-411.

5. B.D. Coleman and M.L. Hodgdon, On shear bands in ductile materials, Arch. Rational Mech. Anal. 90 (1985), 219-247.

6. C.M. Dafermos and L. Hsiao, Global smooth thermomechanical processes in one-dimensional nonlinear thermoviscoelasticity, J. Nonlinear Anal. 6 (1982), 435-454.

7. - Adiabatic shearing of incompressible fluids with temperature dependent viscosity, Quart. Appl. Math. 41 (1983), 45-58.

8. A. Friedman, Partial differential equations of parabolic type, Prentice Hall, Englewood Cliffs, New Jersey, 1964.

9. O.A. Ladyženskaja, Y.A. Solonnikov and N.N. Ural'ceva, Linear and quasilinear equations of parabolic type, (Transl. from Russian by S. Smith), Amer. Math. Soc., Providence, Rhode Island, 1968.

10. J. Leray and J. Schauder, Topologie et eq́uations fonctionelles, Ann. Sci. École Norm. Sup. 51 (1934), 45-78.

11. A. Needleman, Material rate dependence and mesh sensitivity in localization problems, Comput. Methods Appl. Mech. Engrg. 67 (1988), 69-85.

12. J.A. Nohel, R.L. Pego and A.E. Tzavaras, Stability of discontinuous steady states in shearing motions of a non-Newtonian fluid, Proc. Roy. Soc. Edinburgh Sect. A 115 (1990), 39-59.

13. M.H. Protter and H.F. Weinberger, Maximum principles in differential equations, Prentice Hall, Englewood Cliffs, New Jersey, 1967.

14. M. Renardy, W. Hrusa and J. Nohel, Mathematical problems in viscoelasticity, Pitman Monographs Surveys Pure Appl. Math., Vol. 35, Longman Sci. Tech., Essex, England, 1987.

15. T.G. Shawki and R.J. Clifton, Shear band formation in thermal viscoplastic materials, Mech. Mater. 8 (1989), 13-43.

16. A.E. Tzavaras, Shear band formation for materials exhibiting thermal softening, strain hardening and strain-rate sensitivity, Ph.D. Thesis, Brown University, Providence, 1985.

17. - Shearing of materials exhibiting thermal softening or temperature dependent viscosity, Quart. Appl. Math. 44 (1986), 1-12.

18. - Plastic shearing of materials exhibiting strain hardening or strain softening, Arch. Rational Mech. Anal. 94 (1986), 39-58.

19. - Effect of thermal softening in shearing of strain-rate dependent materials, Arch. Rational Mech. Anal. 99 (1987), 349-374.

20. T.W. Wright and J.W. Walter, On stress collapse in adiabatic shear bands, J. Mech. Phys. Solids 35 (1988), 701-720. 
21. F.H. Wu and L.B. Freund, Deformation trapping due to thermoplastic instability in one-dimensional wave propagation, J. Mech. Phys. Solids 32 (1984), 119-132.

Center for the Mathematical Sciences and Department of Mathematics, University OF Wisconsin-MADISON, MADison, WI 53706 